\title{
Frames in cofibration categories
}

\author{
Karol Szumiło 1
}

Received: 8 May 2016 / Accepted: 21 June 2016 / Published online: 13 July 2016

(C) Tbilisi Centre for Mathematical Sciences 2016

\begin{abstract}
We introduce the quasicategory of frames of a cofibration category, i.e. a new model of the $(\infty, 1)$-category associated with a cofibration category.
\end{abstract}

Keywords Homotopy theory · Homotopy colimit · Quasicategory ·

Cofibration category

\section{Introduction}

There are a number of ways of constructing a quasicategory associated with a cofibration category. For example one could take the derived homotopy coherent nerve [4] of its hammock localization [5,6] or apply the derived functor of the Quillen functor $i_{1}^{*}$ of [11] to its classification diagram [15]. Of course these constructions only depend on the weak equivalences and not on cofibrations.

In this paper we introduce a new one called the quasicategory of frames of a cofibration category. It has a number of convenient features compared to the constructions above. It directly yields a quasicategory so that no fibrant replacement in the Joyal model structure (or any of related model categories) is necessary. It does not rely on simplicial enrichment. It takes advantage of the structure of a cofibration category in such a way that homotopy colimits constructed using methods of homotopical algebra can be quite directly translated into quasicategorical colimits.

Communicated by Steve Wilson.

\footnotetext{
$凶 \quad$ Karol Szumiło

kszumilo@uwo.ca

1 Department of Mathematics, University of Western Ontario, 1151 Richmond Street, London, ON N6A 3K7, Canada
} 
This paper is the second in the series of three that summarize the results of the author's thesis [19] (see also [20] for a slightly edited version). The first one [21] constructs a fibration category of cofibration categories which provides a convenient framework for the homotopy theory of cofibration categories. In particular, the notion of a fibration of cofibration categories introduced there is a crucial tool in the present paper. The third one [22] shows that it is possible to reconstruct a cofibration category from a cocomplete quasicategory in a way that establishes an equivalence between the homotopy theory of cofibration categories and the homotopy theory of cocomplete quasicategories. Moreover, in a paper joint with Chris Kapulkin [13] we prove that the quasicategory of frames models the simplicial localization of a cofibration category.

As an example of an application of this construction, it can be shown that the simplicial localization of any categorical model of dependent type theory is a locally cartesian closed quasicategory [12]. This problem has proven difficult when working with known models of simplicial localization. However, every categorical model of type theory is a fibration category [1, Theorem 3.2.5] and hence, by results of the present paper, its localization is a quasicategory with finite limits and our methods can also be used to prove that it is locally cartesian closed. This result can be seen as a step towards describing internal languages of higher categories.

We start with Sect. 1 where we prove a number of preliminary results about diagrams in cofibration categories and fibrations between fibration categories. This section builds directly on [21]. In Sect. 2 we construct a functor from cofibration categories to cocomplete quasicategories. To each cofibration category $\mathcal{C}$ we associate a nerve-like simplicial set denoted by $\mathrm{N}_{\mathrm{f}} \mathcal{C}$ and called the quasicategory of frames in $\mathcal{C}$ (the letter $\mathrm{f}$ in $\mathrm{N}_{\mathrm{f}}$ stands either for frames since those are the objects in $\mathrm{N}_{\mathrm{f}} \mathcal{C}$ or for fractions since the morphisms in $\mathrm{N}_{\mathrm{f}} \mathcal{C}$ are certain generalizations of left fractions). In Sect. 3 we show that $\mathrm{N}_{\mathrm{f}} \mathcal{C}$ is a quasicategory an in Sects. 4 and 5 that it is cocomplete.

Our results are parametrized by a regular cardinal $\kappa$. We fix such $\kappa$ and show that if $\mathcal{C}$ is a $\kappa$-cocomplete cofibration category, then $\mathrm{N}_{\mathrm{f}} \mathcal{C}$ is a $\kappa$-cocomplete quasicategory. In Sects. 2 and 3, to simplify the notation, we take $\kappa=\aleph_{0}$ since the results and methods sections do not depend on $\kappa$. However, the discussion of cocompleteness splits into two cases. The (easier) case of $\kappa>\aleph_{0}$ is dealt with in Sect. 4 and the case of $\kappa=\aleph_{0}$ in Sect. 5 .

\section{Cofibration categories of diagrams}

Our results are based on the techniques of [21] and we start by summarizing the contents of this paper. The central notion is that of cofibration categories which are slightly modified duals of Brown's categories of fibrant objects [3].

Definition 1.1 [21, Definition 1.1] A cofibration category is a category $\mathcal{C}$ equipped with two subcategories: the subcategory of weak equivalences (denoted by $\stackrel{\sim}{\rightarrow}$ ) and the subcategory of cofibrations (denoted by $\longmapsto$ ) such that the following axioms are satisfied (here, an acyclic cofibration is a morphism that is both a weak equivalence and a cofibration).

(C0) Weak equivalences satisfy the 2 -out-of-6 property, i.e. if 


$$
W \stackrel{f}{\longrightarrow} X \stackrel{g}{\longrightarrow} Y \stackrel{h}{\longrightarrow} Z
$$

are morphisms of $\mathcal{C}$ such that both $g f$ and $h g$ are weak equivalences, then so are $f, g$ and $h$ (and thus also $h g f$ ).

(C1) Every isomorphism of $\mathcal{C}$ is an acyclic cofibration.

(C2) An initial object exists in $\mathcal{C}$.

(C3) Every object $X$ of $\mathcal{C}$ is cofibrant, i.e. if 0 is the initial object of $\mathcal{C}$, then the unique morphism $0 \rightarrow X$ is a cofibration.

(C4) Cofibrations are stable under pushouts along arbitrary morphisms of $\mathcal{C}$ (in particular these pushouts exist in $\mathcal{C}$ ). Acyclic cofibrations are stable under pushouts along arbitrary morphisms of $\mathcal{C}$.

(C5) Every morphism of $\mathcal{C}$ factors as a composite of a cofibration followed by a weak equivalence.

(C6) Cofibrations are stable under sequential colimits, i.e. given a sequence of cofibrations

$$
A_{0} \longmapsto A_{1} \longmapsto A_{2} \longleftrightarrow \cdots
$$

its colimit $A_{\infty}$ exists and the induced morphism $A_{0} \rightarrow A_{\infty}$ is a cofibration. Acyclic cofibrations are stable under sequential colimits.

(C7- $\kappa)$ Coproducts of $\kappa$-small families of objects exist. Cofibrations and acyclic cofibrations are stable under $\kappa$-small coproducts.

The last two axioms are optional. If we drop them, then cofibration categories can be considered as models of finitely cocomplete homotopy theories. If we include (C6) and $(\mathrm{C} 7-\kappa)$ for a fixed regular cardinal $\kappa>\aleph_{0}$, we obtain models of $\kappa$-cocomplete homotopy theories, we call them (homotopy) $\kappa$-cocomplete cofibration categories. For $\kappa=\aleph_{0}$ the name (homotopy) $\aleph_{0}$-cocomplete cofibration category will refer to a cofibration category satisfying the axioms $(\mathrm{C} 0-5)$. The definition readily dualizes to yield fibration categories which are models of finitely complete homotopy theories or $\kappa$-complete homotopy theories depending on the choice of axioms.

The main result of [21] establishes the homotopy theory of cofibration categories in the form of a fibration category. We recall the prerequisite definitions before stating the theorem.

Definition 1.2 A functor $F: \mathcal{C} \rightarrow \mathcal{D}$ between cofibration categories is exact if it preserves cofibrations, acyclic cofibrations, initial objects and pushouts along cofibrations.

If $\mathcal{C}$ and $\mathcal{D}$ are $\kappa$-cocomplete, then $F$ is $\kappa$-cocontinuous if, in addition, it preserves colimits of sequences of cofibrations and $\kappa$-small coproducts.

The category of (small) $\kappa$-cocomplete cofibration categories and $\kappa$-cocontinuous functors will be denoted by CofCat ${ }_{\kappa}$. It is equipped with classes of weak equivalences and fibrations as defined below.

Definition 1.3 An exact functor $F: \mathcal{C} \rightarrow \mathcal{D}$ is a weak equivalence if it induces an equivalence $\mathrm{Ho} \mathcal{C} \rightarrow \mathrm{Ho} \mathcal{D}$. 
Definition 1.4 [21, Definition 2.3] Let $P: \mathcal{E} \rightarrow \mathcal{D}$ be an exact functor of cofibration categories.

(1) $P$ is an isofibration if for every object $A \in \mathcal{E}$ and an isomorphism $g: P A \rightarrow Y$ there is an isomorphism $f: A \rightarrow B$ such that $P f=g$.

(2) It is said to satisfy the lifting property for factorizations if for any morphism $f: A \rightarrow B$ of $\mathcal{E}$ and a factorization

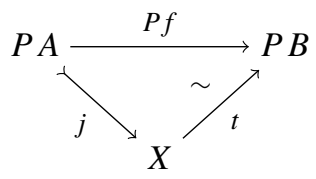

there exists a factorization

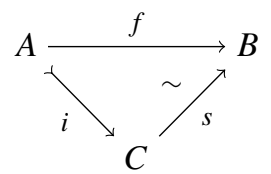

such that $P i=j$ and $P s=t$ (in particular, $P C=X$ ).

(3) It has the lifting property for pseudofactorizations if for any morphism $f: A \rightarrow B$ of $\mathcal{E}$ and a diagram

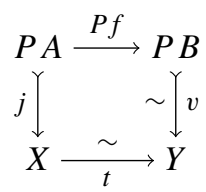

there exists a diagram

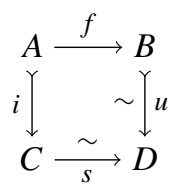

such that $P i=j, P s=t$ and $P u=v$ (in particular, $P C=X$ and $P D=Y$ ).

(4) We say that $P$ is a fibration if it is an isofibration and satisfies the lifting properties for factorizations and pseudofactorizations.

Theorem 1.5 [21, Theorem 2.8] The category CofCat $_{\kappa}$ of small $\kappa$-cocomplete cofibration categories with weak equivalences and fibrations as above is a fibration category.

In the remainder of this section we will introduce a general technique of constructing fibrations of cofibration categories which relies on the notions of direct categories and Reedy cofibrations. We will not discuss the basic theory of Reedy cofibrations since it is already well covered in the literature. A good general reference is [17] which is written from the perspective of Reedy categories and model categories. The theory of diagrams over general Reedy categories requires using both colimits and limits. Thus 
in the case of cofibration categories we have to restrict attention to a special class of Reedy categories called direct categories where colimits suffice. Specific results concerning Reedy cofibrations in cofibration categories are explained in [14] from where we will cite a few most relevant to the purpose of this paper.

Definition 1.6 (1) A category $I$ is direct if it admits a functor deg: $I \rightarrow \mathbb{N}$ that reflects identities, i.e. if $\varphi: i \rightarrow j$ is a morphism of $I$ such that $\operatorname{deg} i=\operatorname{deg} j$, then $i=j$ and $\varphi=\mathrm{id}_{i}$ (we consider $\mathbb{N}$ as a poset with its standard order).

(2) For a direct category $I$ and $i \in I$, the latching category at $i$ is the full subcategory of the slice $I \downarrow i$ on all objects except for $\mathrm{id}_{i}$. It is denoted by $\partial(I \downarrow i)$.

(3) Let $X: I \rightarrow \mathcal{C}$ be a diagram in some category and $i \in I$. The latching object of $X$ at $i$ is the colimit of the composite diagram

$$
\partial(I \downarrow i) \rightarrow I \rightarrow \mathcal{C}
$$

where $\partial(I \downarrow i) \rightarrow I$ is the forgetful functor sending a morphism of $I$ (i.e. an object of $\partial(I \downarrow i))$ to its source. The latching object (if it exists) is denoted by $L_{i} X$ and comes with a canonical latching morphism $L_{i} X \rightarrow X_{i}$ induced by the inclusion $\partial(I \downarrow i) \rightarrow I \downarrow i$.

(4) Let $\mathcal{C}$ be a cofibration category. A diagram $X: I \rightarrow \mathcal{C}$ is Reedy cofibrant if for all $i \in I$ the latching object of $X$ at $i$ exists and the latching morphism $L_{i} X \rightarrow X_{i}$ is a cofibration.

(5) Let $f: X \rightarrow Y$ be a morphism of Reedy cofibrant diagrams $I \rightarrow \mathcal{C}$. It is called a Reedy cofibration if for all $i \in I$ the induced morphism

$$
X_{i} \amalg_{L_{i} X} L_{i} Y \rightarrow Y_{i}
$$

is a cofibration (observe that this pushout exists since $X$ is Reedy cofibrant).

The main purpose of this section is to construct certain cofibration categories of diagrams and establish some practical criteria for verifying that particular functors between them are weak equivalences or fibrations.

Proposition 1.7 Let $\mathcal{C}$ be a cofibration category and $J$ a homotopical direct category with finite latching categories.

(1) The category $\mathcal{C}_{\mathrm{R}}^{J}$ of homotopical Reedy cofibrant diagrams with levelwise weak equivalences and Reedy cofibrations is a cofibration category.

(2) The category $\mathcal{C}^{J}$ of all homotopical diagrams with levelwise weak equivalences and levelwise cofibrations is a cofibration category.

(3) The inclusion functor $\mathcal{C}_{\mathrm{R}}^{J} \hookrightarrow \mathcal{C}^{J}$ is a weak equivalence.

Proof (1) [14, Theorem 9.3.8 (1a)]

(2) $[14$, Theorem $9.3 .8(1 \mathrm{~b})]$

(3) The inclusion functor satisfies the approximation properties of [21, Proposition 2.2] as follows from Lemma 1.9 (1) (in fact, from its standard special case of $\mathcal{D}=[0]$ and $I=\varnothing)$. 
The crucial step in the proof of the above proposition is the construction of factorizations. In Lemma 1.9 we revisit that construction in order to prove a more general version which will be a key technical tool in many arguments of this paper.

A homotopical functor $f: I \rightarrow J$ is a homotopy equivalence if there is a homotopical functor $g: J \rightarrow I$ such that $g f$ is weakly equivalent to $\mathrm{id}_{I}$ and $f g$ is weakly equivalent to id ${ }_{J}$ (where "weakly equivalent" means "connected by a zig-zag of natural weak equivalences").

Lemma 1.8 Let $\mathcal{C}$ be a cofibration category and $f: I \rightarrow J$ a homotopical functor where $I$ and $J$ are homotopical direct categories with finite latching categories.

(1) The induced functor $f^{*}: \mathcal{C}^{J} \rightarrow \mathcal{C}^{I}$ is exact.

(2) If $f$ is a homotopy equivalence, then $f^{*}: \mathcal{C}^{J} \rightarrow \mathcal{C}^{I}$ is a weak equivalence of cofibration categories.

(3) If $f$ is a homotopy equivalence and induces an exact functor $f^{*}: \mathcal{C}_{\mathrm{R}}^{J} \rightarrow \mathcal{C}_{\mathrm{R}}^{I}$, then it is also a weak equivalence.

Proof The functor $f^{*}$ is clearly exact with respect to the levelwise structures and it is a homotopy equivalence when $f$ is.

For the last statement, consider the commutative square of exact functors

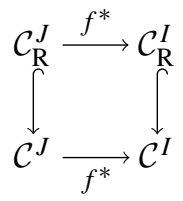

the vertical maps are weak equivalences by Proposition 1.7 so the conclusion follows by 2 -out-of- 3 .

The utility of direct categories comes from the fact that it is easy to construct diagrams and morphisms of diagrams inductively. For our purposes it will be most convenient to state this in terms of sieves. A functor $I \rightarrow J$ is called a sieve if it is an inclusion of a full downwards closed subcategory, i.e. if it is injective on objects, fully faithful and if $i \rightarrow j$ is a morphism of $J$ such that $j \in I$, then $i \in I$. If $I$ and $J$ are homotopical categories, we will further assume as a part of the definition of a sieve $I \rightarrow J$ that it preserves and reflects weak equivalences, i.e. a morphism of $I$ is a weak equivalence if and only if its image in $J$ is.

The first part of the next lemma generalizes the standard construction of factorizations into Reedy cofibrations followed by weak equivalences. It says that given a morphism of diagrams $J \rightarrow \mathcal{C}$ and compatible factorizations of its restriction along a sieve $I \hookrightarrow J$ and its image under a fibration $P: \mathcal{C} \rightarrow \mathcal{D}$, there is a factorization of the original morphism compatible with both of them. The other two parts say the same for lifts for pseudofactorizations and for cofibrations (when $P$ is an acyclic fibration as in [21, Proposition 2.5]).

Lemma 1.9 Let $P: \mathcal{C} \rightarrow \mathcal{D}$ be a fibration between cofibration categories. Let $J$ be a homotopical direct category with finite latching categories and $I \hookrightarrow J$ a sieve.

(1) Let $f: X \rightarrow Y$ be a morphism in $\mathcal{C}^{J}$. If $X$ is Reedy cofibrant, 


$$
P X \stackrel{k_{P}}{\longrightarrow} \widetilde{Y}_{P} \stackrel{s_{P}}{\sim} P Y \quad \text { and } \quad X\left|I \stackrel{k_{I}}{\longleftrightarrow} \widetilde{Y}_{I} \stackrel{s_{I}}{\sim} Y\right| I
$$

are factorizations of $P f$ and $f \mid I$ into Reedy cofibrations followed by weak equivalences such that $P k_{I}=k_{P} \mid I$ and $P s_{I}=s_{P} \mid I$ (in particular, $P \widetilde{Y}_{I}=\widetilde{Y}_{P} \mid I$ ), then there is a factorization

$$
X \stackrel{k}{\longrightarrow} \widetilde{Y} \stackrel{s}{\sim} Y
$$

of $f$ into a Reedy cofibration followed by a weak equivalence such that $P k=k_{P}$, $k \mid I=k_{I}, P s=s_{P}$ and $s \mid I=s_{I}$ (in particular, $P \widetilde{Y}=\widetilde{Y}_{P}$ and $\widetilde{Y} \mid I=\widetilde{Y}_{I}$ ).

(2) Let $f: X \rightarrow Y$ be a morphism in $\mathcal{C}^{J}$. If both $X$ and $Y$ are Reedy cofibrant,

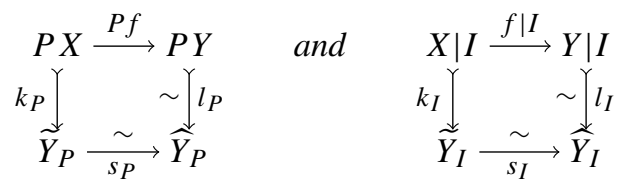

are pseudofactorizations of $P f$ and $f \mid I$ such that $P k=k_{p}, k \mid I=k_{I}, P l=l_{P}$, $l \mid I=l_{I}, P s=s_{P}$ and $s \mid I=s_{I}$ (in particular, $P \widetilde{Y}_{I}=\widetilde{Y}_{P} \mid I$ and $P \widehat{Y}_{I}=\widehat{Y}_{P} \mid I$ ), then there is a pseudofactorization

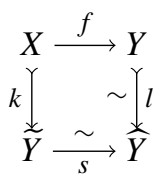

such that $P k=k_{P}, k\left|I=k_{I}, P l=l_{P}, l\right| I=l_{I}, P s=s_{P}$ and $s \mid I=s_{I}$ (in particular, $P \widetilde{Y}=\widetilde{Y}_{P}, \widetilde{Y} \mid I=\widetilde{Y}_{I}, P \widehat{Y}=\hat{Y}_{P}$ and $\left.\widehat{Y} \mid I=\widehat{Y}_{I}\right)$.

(3) If $P$ is acyclic, $X$ is a Reedy cofibrant diagram in $\mathcal{C}^{J}$ and

$$
P X \stackrel{k_{P}}{\longrightarrow} Z_{P} \quad X \mid I \stackrel{k_{I}}{\longmapsto} Z_{I}
$$

are Reedy cofibrations such that $P k_{I}=k_{P} \mid I$, then there exists a Reedy cofibration

$$
X \stackrel{k}{\longrightarrow} Z
$$

such that $P k=k_{P}$ and $k \mid I=k_{I}$ (in particular, $P Z=Z_{P}$ and $Z \mid I=Z_{I}$ ).

Proof The proofs of three parts are similar to each other so we only provide the first one. (the second one uses the lifting property for pseudofactorizations and the third one uses the lifting property of [21, Proposition 2.5]).

It suffices to extend the factorization $f \mid I=s_{I} k_{I}$ over an object $j \in J \backslash I$ of a minimal degree. Then the statement will follow by an induction over the degree.

By the minimality of the degree of $j$, Reedy cofibrancy of $X$ and since $I \hookrightarrow J$ is a sieve the latching objects $L_{j} X$ and $L_{j} \widetilde{Y}_{I}$ exist. Moreover, the induced functor of latching categories $\partial(I \downarrow j) \rightarrow \partial(J \downarrow j)$ is an isomorphism. Thus $P$ sends the morphism $X_{j} \amalg_{L_{j} X} L_{j} \widetilde{Y}_{I} \rightarrow Y_{j}$ to the analogous morphism $P X_{j} \amalg_{L_{j} P X} P \widetilde{Y}_{I} \rightarrow P Y_{j}$. The 
latter factors as

$$
P X_{j} \amalg_{L_{j} P X} P \widetilde{Y}_{I} \longmapsto\left(\widetilde{Y}_{P}\right)_{j} \stackrel{\sim}{\rightarrow} P Y_{j}
$$

and since $P$ is a fibration we can lift this to a factorization of the former as

$$
X_{j} \amalg_{L_{j} X} L_{j} \tilde{Y}_{I} \longmapsto \widetilde{Y}_{j} \stackrel{\sim}{\rightarrow} Y_{j}
$$

This extends the factorization $f \mid I=s_{I} k_{I}$ over $j$.

The resulting diagram $\widetilde{Y}$ is homotopical since it is weakly equivalent to homotopical $Y$.

The most typical examples of fibrations are restrictions along sieves.

Lemma 1.10 Let $\mathcal{C}$ be a cofibration category. If $I$ and $J$ are homotopical direct categories with finite latching categories and $f: I \rightarrow J$ a homotopical functor such that for every $i \in I$ the induced functor of the latching categories $\partial(I \downarrow i) \rightarrow \partial(J \downarrow f i)$ is an isomorphism, then the functor $f^{*}: \mathcal{C}^{J} \rightarrow \mathcal{C}^{I}$ induces a functor $f^{*}: \mathcal{C}_{\mathrm{R}}^{J} \rightarrow \mathcal{C}_{\mathrm{R}}^{I}$ which is exact.

Moreover, if $f$ is a sieve, then $f^{*}$ is a fibration.

Proof If $f$ induces isomorphisms of the latching categories, then $f^{*}$ preserves Reedy cofibrations (and, in particular, Reedy cofibrant diagrams). It also preserves weak equivalences and colimits that exist in $\mathcal{C}_{\mathrm{R}}^{J}$ so it is exact.

If $f$ is a sieve, then it satisfies the exactness criterion above. Moreover, $f^{*}$ is a fibration by parts (1) and (2) of Lemma 1.9.

The next few lemmas establish some connections between sieves and fibrations which are reminiscent of classical homotopical algebra if we think of sieves as "cofibrations" and sieves $I \hookrightarrow J$ inducing weak equivalences $\mathcal{C}_{\mathrm{R}}^{J} \rightarrow \mathcal{C}_{\mathrm{R}}^{I}$ as "acyclic cofibrations". This does not quite fit into the classical picture since such "cofibrations" do not really belong to the same category as the fibrations. The situation bears some resemblance to the "pushout product property" of a simplicial model category $\mathcal{M}$ (see e.g. [8, Definition 4.2.18]), but is different. In the present context it is essential that Reedy cofibrant diagrams can be seen as "morphisms" from direct categories to cofibration categories, while in the context of the pushout product property there is usually no meaningful notion of a morphism from a simplicial set to an object of $\mathcal{M}$.

Lemma 1.11 Let $f: I \hookrightarrow J$ be a sieve between homotopical direct categories with finite latching categories and $P: \mathcal{C} \rightarrow \mathcal{D}$ a fibration of cofibration categories. Then the induced exact functor $\left(f^{*}, P\right): \mathcal{C}_{\mathrm{R}}^{J} \rightarrow \mathcal{C}_{\mathrm{R}}^{I} \times{ }_{\mathcal{D}_{\mathrm{R}}^{I}} \mathcal{D}_{\mathrm{R}}^{J}$

(1) is a fibration,

(2) is an acyclic fibration provided that $P$ is acyclic,

(3) is an acyclic fibration provided that both $f^{*}: \mathcal{C}_{\mathrm{R}}^{J} \rightarrow \mathcal{C}_{\mathrm{R}}^{I}$ and $f^{*}: \mathcal{D}_{\mathrm{R}}^{J} \rightarrow \mathcal{D}_{\mathrm{R}}^{I}$ are weak equivalences.

Proof First observe that the pullback in question exists since $f^{*}$ is a fibration by Lemma 1.10. 
(1) This follows by parts (1) and (2) of Lemma 1.9.

(2) This follows by (1) above and part (3) of Lemma 1.9.

(2) This follows by (1) above and a diagram chase using the fact that acyclic fibrations are closed under pullbacks.

Lemma 1.12 If $\mathcal{C}$ is a cofibration category,

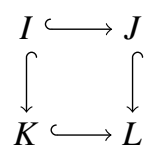

is a pushout square of homotopical direct categories with finite latching categories and both $I \hookrightarrow J$ and $I \hookrightarrow K$ are sieves, then the resulting square<smiles>[R][Z1]1=[R][R]1</smiles>

is a pullback of cofibration categories.

Proof By the construction of pullbacks of cofibration categories it will suffice to verify that a morphism of diagrams over $L$ is a Reedy cofibration if and only if it is one when restricted to both $J$ and $K$. For this it will be enough to observe that both $J \hookrightarrow L$ and $K \hookrightarrow L$ are sieves and hence for an object $l \in L$ we have either $l \in J$ and then $\partial(J \downarrow l) \rightarrow \partial(L \downarrow l)$ is an isomorphism or $l \in K$ and then $\partial(K \downarrow l) \rightarrow \partial(L \downarrow l)$ is an isomorphism.

Let $f: I \rightarrow J$ be a homotopical functor of homotopical direct categories and $F: \mathcal{C} \rightarrow \mathcal{D}$ an exact functor of cofibration categories. We say that $f$ has the Reedy left lifting property with respect to $F$ (or $F$ has the Reedy right lifting property with respect to $f$ ) if every lifting problem

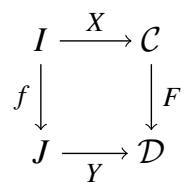

where $X$ and $Y$ are homotopical Reedy cofibrant diagrams has a solution that is also a homotopical Reedy cofibrant diagram.

Lemma 1.13 Let $f: I \hookrightarrow J$ and $g: K \rightarrow L$ be sieves between homotopical direct categories with finite latching categories and $F: \mathcal{C} \rightarrow \mathcal{D}$ an exact functor of cofibration categories. Then there is a natural bijection between Reedy lifting problems (and their solutions) of the forms
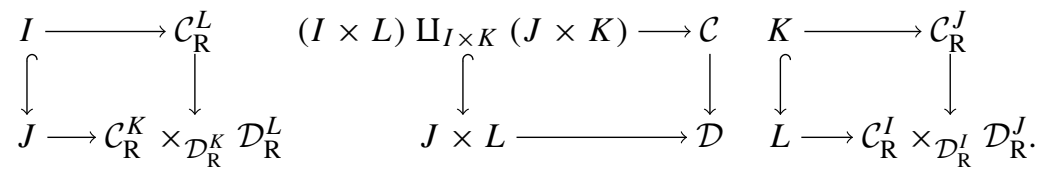
Proof This this will follow from standard adjointness arguments, e.g. as in [10, Proposition D.1.18], if we can verify that a diagram $X: J \times L \rightarrow \mathcal{C}$ is Reedy cofibrant if and only if the corresponding diagram $\widetilde{X}: J \rightarrow \mathcal{C}^{L}$ is Reedy cofibrant as a diagram $J \rightarrow \mathcal{C}_{\mathrm{R}}^{L}$.

First, assume that $X$ is Reedy cofibrant. For $j \in J$, the diagrams $\widetilde{X}_{j}, L_{j} \widetilde{X}: L \rightarrow \mathcal{C}$ can be computed as the left Kan extensions of (restrictions of) $X$ along the projections $(J \downarrow j) \times L \rightarrow L$ and $\partial(J \downarrow j) \times L \rightarrow L$. These Kan extensions exist and are Reedy cofibrant by [14, Theorem 9.4.3 (1)]. Moreover, it follows from [14, Theorem 9.4.1 (1)] that the induced morphism $L_{j} \widetilde{X} \rightarrow \widetilde{X}_{j}$ is a Reedy cofibration since the inclusion $\partial(J \downarrow j) \times L \hookrightarrow(J \downarrow j) \times L$ is a sieve. Thus $\tilde{X}$ is a Reedy cofibrant $\operatorname{diagram} J \rightarrow \mathcal{C}_{\mathrm{R}}^{L}$.

Conversely, assume that $\widetilde{X}$ is a Reedy cofibrant diagram $J \rightarrow \mathcal{C}_{\mathrm{R}}^{L}$. For all $j \in J$ and $l \in L$, we need to verify that the latching object $L_{j, l} X$ exists and the latching morphism $L_{j, l} X \rightarrow X_{j, l}$ is a cofibration. Proceeding by induction, we may assume that this true for all objects of $J \times L$ lying strictly below $(j, l)$. This implies that the composite diagram

$$
\partial(J \times L \downarrow(j, l)) \rightarrow J \times L \rightarrow \mathcal{C}
$$

is Reedy cofibrant and hence $L_{j, l} X$ exists. Moreover, there is a pushout square of direct categories

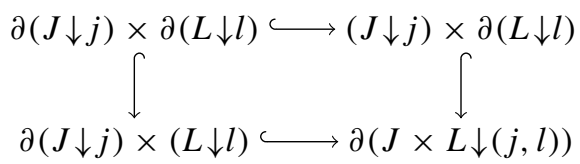

(where all maps are sieves) which implies that $L_{j, l}$ can also be computed as the pushout

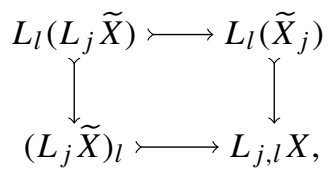

i.e. $L_{j, l} X$ coincides with the relative latching object of the morphism $L_{j} \widetilde{X} \rightarrow \widetilde{X}_{j}$ at $l$. Hence $L_{j, l} X \rightarrow X_{j, l}$ is a cofibration since $\widetilde{X}$ is Reedy cofibrant.

Lemma 1.14 Let $P: \mathcal{C} \rightarrow \mathcal{D}$ be a fibration of cofibration categories. The following are equivalent:

(1) $P$ is acyclic,

(2) $P$ has the Reedy right lifting property with respect to all sieves between direct homotopical categories with finite latching categories,

(3) $P$ has the Reedy right lifting property with respect to [0] $\hookrightarrow[1]$ and [1] $\hookrightarrow \widehat{[1]}$.

Proof If $P$ is acyclic, then it has the Reedy right lifting property with respect to all sieves between homotopical direct categories with finite latching categories by Lemma 1.9 (3), in particular, with respect to [0] $\hookrightarrow[1]$ and [1] $\hookrightarrow \widehat{[1]}$. 
Conversely, by Lemma 1.11 it suffices to see that if $P$ has the Reedy right lifting property with respect to [0] $\hookrightarrow[1]$ and [1] $\hookrightarrow \widehat{[1]}$, then it satisfies (App1) and has the right lifting property in $\overline{\mathrm{CofCat}}$ with respect to the inclusion of [0] into

$$
0 \longmapsto 1 \text {. }
$$

The latter is equivalent to the Reedy right lifting property with respect to [0] $\hookrightarrow[1]$. To see that the Reedy right lifting property with respect to [1] $\hookrightarrow \widehat{[1]}$ implies (App1) take a morphism $f: X \rightarrow Y$ in $\mathcal{C}$ such that $P f$ is a weak equivalence. Factor $f$ as

$$
X \stackrel{j}{\longrightarrow} \widetilde{Y} \stackrel{\sim}{\longrightarrow} Y .
$$

Then $P j$ is a weak equivalence by 2 -out-of- 3 and hence so is $j$ by the Reedy right lifting property with respect to [1] $\hookrightarrow \widehat{[1]}$. Thus $f$ is a weak equivalence, too.

Lemma 1.15 If a sieve $f: I \rightarrow J$ between homotopical direct categories has the Reedy left lifting property with respect to all fibrations of cofibration categories, then for every cofibration category $\mathcal{C}$ the induced functor $f^{*}: \mathcal{C}_{\mathrm{R}}^{J} \rightarrow \mathcal{C}_{\mathrm{R}}^{I}$ is an acyclic fibration.

Proof Since $f$ is a sieve, $f^{*}$ is a fibration by Lemma 1.10 . Thus, by Lemma 1.14 , it will suffice to check that $f^{*}$ has the Reedy right lifting property with respect to $[0] \hookrightarrow[1]$ and [1] $\hookrightarrow \widehat{[1]}$. These are equivalent to the Reedy right lifting property of $\mathcal{C}_{\mathrm{R}}^{[1]} \rightarrow \mathcal{C}_{\mathrm{R}}^{[0]}$ and $\mathcal{C}_{\mathrm{R}}^{[\widehat{1]}} \rightarrow \mathcal{C}_{\mathrm{R}}^{[1]}$ with respect to $I \hookrightarrow J$ by Lemma 1.13 .

\section{Quasicategories of frames}

Before introducing quasicategories of frames we need to explain a preliminary construction which will play an essential role in the remainder of this paper. It depends on properties of direct and homotopical categories discussed in Sect. 1.

Let $\Delta_{\sharp}$ denote the subcategory of injective maps in $\Delta$ and let $J$ be a homotopical category. We construct a direct homotopical category $D J$ and a homotopical functor $p_{J}: D J \rightarrow J$ as follows. The underlying category of $D J$ is the comma category $\Delta_{\sharp \downarrow} J$, i.e. objects are all functors $[m] \rightarrow J$ for all $m$ and a morphism from $x:[m] \rightarrow$ $J$ to $y:[n] \rightarrow J$ is an injective order preserving $\operatorname{map} \varphi:[m] \hookrightarrow[n]$ such that $x=y \varphi$. The functor $p_{J}: \Delta_{\sharp \downarrow} J \rightarrow J$ (sometimes called the last vertex projection) is defined by sending $x:[m] \rightarrow J$ to $x_{m}$ and a morphism $\varphi$ as above to the induced morphism $x_{m}=y_{\varphi(m)} \rightarrow y_{n}$. The weak equivalences in $D J$ are created by $p_{J}$. Then $D J$ is homotopical category, $p_{J}$ is a homotopical functor and $D J$ is also direct (by setting the degree of $[m] \rightarrow J$ to $m$ ). We can think of $D J$ as a direct approximation to $J$. Observe that $D$ is a functor from homotopical categories to homotopical categories and that $D J$ has a non-trivial homotopical structure even if $J$ has the trivial one (unless $J$ is empty). This construction has multiple motivations which will be given right after the definition of quasicategories of frames below.

First, we need to verify that Reedy cofibrant diagrams over $D J$ are well behaved with respect to homotopical functors $I \rightarrow J$. If $f$ is such a functor we will abbreviate the induced functor $(D f)^{*}: \mathcal{C}_{\mathrm{R}}^{D J} \rightarrow \mathcal{C}_{\mathrm{R}}^{D I}$ to $f^{*}$ to simplify the notation. Recall 
that $\mathcal{C}_{\mathrm{R}}^{D J}$ refers to the cofibration category of homotopical Reedy cofibrant diagrams $D J \rightarrow \mathcal{C}$ with levelwise weak equivalences and Reedy cofibrations which exists by [14, Theorem 9.3 .8 (1a)].

Lemma 2.1 Let $\mathcal{C}$ be a cofibration category. If $f: I \rightarrow J$ is a homotopical functor of small homotopical categories, then the induced functor $f^{*}: \mathcal{C}_{\mathrm{R}}^{D J} \rightarrow \mathcal{C}_{\mathrm{R}}^{D I}$ is exact. If $f$ is injective on objects and faithful, then $f^{*}$ is a fibration.

Proof For every $x:[m] \rightarrow I$, the induced functor of latching categories $\partial(D I \downarrow x) \rightarrow$ $\partial(D J \downarrow f x)$ is an isomorphism since both are essentially copies of $\partial\left(\Delta_{\sharp} \downarrow[m]\right)$. Moreover, if $f$ is injective on objects and faithful, then $D f$ is a sieve. Thus both statements follow from Lemma 1.10 .

For a cofibration category $\mathcal{C}$ we define the quasicategory of frames in $\mathcal{C}$ as a simplicial set denoted by $\mathrm{N}_{\mathrm{f}} \mathcal{C}$ where $\left(\mathrm{N}_{\mathrm{f}} \mathcal{C}\right)_{m}$ is the set of all homotopical Reedy cofibrant diagrams $D[m] \rightarrow \mathcal{C}([m]$ is a homotopical category with only identities as weak equivalences). The simplicial structure is given by functoriality of $D$ (using Lemma 2.1 to see that simplicial operators preserve Reedy cofibrancy). Since exact functors of cofibration categories preserve Reedy cofibrant diagrams, $\mathrm{N}_{\mathrm{f}}$ is a functor from the category of cofibration categories to the category of simplicial sets.

Remark 2.2 As a side note, we point out that this construction can be enhanced as follows. If $\widehat{[n]}$ denotes the homotopical poset $[n]$ with all morphisms as weak equivalences, then the bisimplicial set

$$
[m],[n] \mapsto\{\text { homotopical Reedy cofibrant diagrams } D([m] \times \widehat{[n]}) \rightarrow \mathcal{C}\}
$$

is a complete Segal space with $\mathrm{N}_{\mathrm{f}} \mathcal{C}$ as its 0 th row. This enhancement will be closely analyzed in [13].

This definition can be motivated as follows. First, the objects of $\mathrm{N}_{\mathrm{f}} \mathcal{C}$ are called frames in $\mathcal{C}$. They are counterparts to frames in a model category $\mathcal{M}$, i.e. homotopically constant Reedy cofibrant diagrams $\Delta \rightarrow \mathcal{M}$ which can be used to enrich the homotopy category Ho $\mathcal{M}$ in the homotopy category of simplicial sets as explained in [8, Chapter 5]. In cofibration categories we are forced to replace $\Delta$ by $\Delta_{\sharp}$ since working with $\Delta$ would require referring to the matching objects of cosimplicial objects which are defined as certain limits and hence are not available in a cofibration category. The homotopically constant diagrams over $\Delta_{\sharp}$ are precisely the homotopical diagrams over $D[0]$. Again, one can prove using such frames that the homotopy category $\mathrm{Ho} \mathcal{C}$ is enriched in the category of homotopy types, see [18, Theorems 3.10 and 3.17]. ${ }^{1}$ Our construction can be seen as an alternative way of using frames to enrich $\mathrm{Ho} \mathcal{C}$ in homotopy types, namely, by using the mapping spaces of the quasicategory $\mathrm{N}_{\mathrm{f}} \mathcal{C}$.

The second motivation is that $\mathrm{N}_{\mathrm{f}} \mathcal{C}$ can be seen as an enhancement of the calculus of fractions. Let $\operatorname{Sd}[m]$ denote the poset of non-empty subsets of $m$. It can be seen

1 This result differs from its counterpart for model categories since it uses presimplicial sets (a.k.a. $\Delta$-sets or semisimplicial sets) as models of homotopy types. Presimplicial sets are less well-behaved than simplicial sets, but their homotopy theory is equivalent to that of simplicial sets, see e.g. [16, Proposition 2.1]. 
as the full subcategory of $D[\mathrm{~m}]$ spanned by the non-degenerate simplices of $[\mathrm{m}]$ as explained in more detail on p. 21. Homotopical Reedy cofibrant diagrams over $D[\mathrm{~m}]$ can be seen as resolutions of their restrictions to $\mathrm{Sd}[m]$. Therefore an object of $\mathrm{N}_{\mathrm{f}} \mathcal{C}$ is a resolution of an object of $\mathcal{C}$ and a morphism is a resolution of a diagram of the form

$$
X_{0} \longrightarrow X_{01} \stackrel{\sim}{\longleftarrow} X_{1}
$$

i.e. a left fraction from $X_{0}$ to $X_{1}$. Similarly, a 2-simplex of $\mathrm{N}_{\mathrm{f}} \mathcal{C}$ is a resolution of a diagram of the form

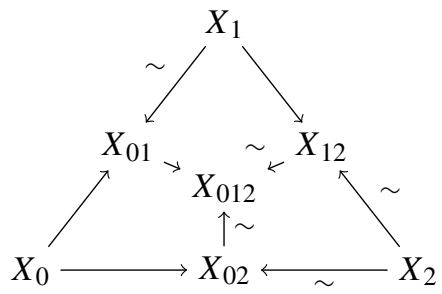

which consists of two fractions going from $X_{0}$ to $X_{1}$ and from $X_{1}$ to $X_{2}$ along with a composite fraction going directly from $X_{0}$ to $X_{2}$. Such diagrams simultaneously encode the composition of left fractions and the notion of equivalence of fractions. Higher simplices encode the higher homotopy of the mapping spaces of $\mathcal{C}$ in a similar manner.

It might be tempting to simplify the definition of $\mathrm{N}_{\mathrm{f}} \mathcal{C}$ by replacing $D[m]$ with $\operatorname{Sd}[m]$. This would not work since functors $\mathrm{Sd}[m] \rightarrow \operatorname{Sd}[n]$ induced by degeneracy operators $[m] \rightarrow[n]$ do not respect Reedy cofibrant diagrams and thus this modification would not even yield a simplicial set.

Here is our main result. It is parametrized by a regular cardinal $\kappa$. In the first two sections we will assume that $\kappa=\aleph_{0}$ to simplify the exposition.

Theorem 2.3 If C is a $\kappa$-cocomplete cofibration category, then $\mathrm{N}_{\mathrm{f}} \mathcal{C}$ is a $\kappa$-cocomplete quasicategory.

Before proceeding with the proof, we will give another version of the $D$ construction. For a simplicial set $K$ we define a homotopical direct category $D K$ as follows. The underlying category of $D K$ is the category of elements of $K$ but only with face operators as morphisms, i.e. objects of $D K$ are all simplices of $K$ and a morphism from $x \in K_{m}$ to $y \in K_{n}$ is an injective order preserving map $\varphi:[m] \hookrightarrow[n]$ such that $x=y \varphi$.

Such a morphism is a generating weak equivalence if $y v$ is a degenerate edge of $K$ where $v:[1] \rightarrow[n]$ is defined by $v(0)=\varphi(m)$ and $v(1)=n$. The generating weak equivalences do not necessarily satisfy the 2-out-of-6 property (they are not even closed under composition in general). Thus we define the subcategory of weak equivalences as the smallest subcategory containing the generating weak equivalences and satisfying the 2-out-of-6 property. Of course, in order to verify that a functor from $D K$ to a homotopical category is homotopical it suffices to check that it sends the generating weak equivalences to weak equivalences. 
This construction is functorial in $K$. Moreover, the next lemma says that if $K$ is the nerve of a category $J$, then $D K$ coincides with $D J$ in the sense of the previous definition.

Lemma 2.4 Let $J$ be a category with the trivial homotopical structure. Then the homotopical categories DJ and DNJ coincide.

Proof The underlying categories of $D J$ and $D N J$ are the same by definition. The generating weak equivalences of $D N J$ are mapped to identities by $p_{J}: D J \rightarrow J$ and hence it suffices to see that every weak equivalence created by $p_{J}$ can be obtained from the generating ones by applying the 2-out-of-6 property. Let $\varphi, \psi \in D J$ and consider a morphism $\varphi \rightarrow \psi$ mapped by $p_{J}$ to an isomorphism $f: x \rightarrow y$ of $J$. Then we have a diagram

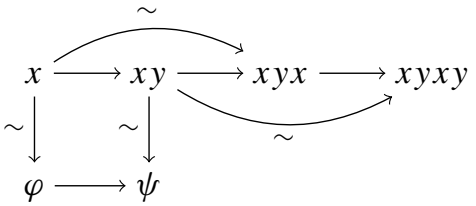

in $D J$ where $x y x y$ denotes the sequence

$$
x \stackrel{f}{\longrightarrow} y \stackrel{f^{-1}}{\longrightarrow} x \stackrel{f}{\longrightarrow} y
$$

and the remaining objects in the first row are its initial segments. The indicated morphisms are generating weak equivalences and hence by 2-out-of-6 $\varphi \rightarrow \psi$ is also a weak equivalence of $D N J$.

Lemma 2.5 The functor $D:$ sSet $\rightarrow$ Cat (i.e. when we disregard the homotopical structures of $\mathrm{DKs}$ ) preserves colimits.

Proof Since $N$ : Cat $\rightarrow$ sSet is fully faithful it reflects colimits (see [2, Proposition 2.2.9]). Thus it will suffice to verify that the composite functor $K \mapsto N D K$ preserves colimits. This follows from the fact that

$$
(N D K)_{m}=\coprod_{\left[j_{0}\right] \hookrightarrow\left[j_{1}\right] \hookrightarrow \cdots \hookrightarrow\left[j_{m}\right]} K_{j_{m}} .
$$

Let $X: D K \rightarrow \mathcal{C}$ be a homotopical Reedy cofibrant diagram. For each simplex $x: \Delta[m] \rightarrow K$ consider the restriction $x^{*} X: D[m] \rightarrow \mathcal{C}$ which is an $m$-simplex of $\mathrm{N}_{\mathrm{f}} \mathcal{C}$ (recall that $x^{*}$ is an abbreviation of $\left.(D x)^{*}\right)$. These simplices fit together to form a simplicial map $K \rightarrow \mathrm{N}_{\mathrm{f}} \mathcal{C}$.

Proposition 2.6 Let $\mathcal{C}$ be a cofibration category and $K$ a simplicial set. The map described above is a natural bijection between

- the set of homotopical Reedy cofibrant diagrams $D K \rightarrow \mathcal{C}$

- and the set of simplicial maps $K \rightarrow \mathrm{N}_{\mathrm{f}} \mathcal{C}$. 
Proof Denote the former set by $R(D K, \mathcal{C})$ and observe that $R(D-, \mathcal{C})$ is a contravariant functor from simplicial sets to sets. The statement says that this functor is representable and the representing object is $\mathrm{N}_{\mathrm{f}} \mathcal{C}$. This will follow if we can verify that if we consider any simplicial set $K$ as a colimit of its simplices, then this colimit is preserved (i.e. carried to a limit) by $R(D-, \mathcal{C})$.

First, note that by Lemma 2.5 the functor $\operatorname{Cat}(D-, \mathcal{C})$ carries colimits to limits. Since $R(D-, \mathcal{C})$ is a subfunctor of $\operatorname{Cat}(D-, \mathcal{C})$ it will suffice to see that a diagram $X: D K \rightarrow \mathcal{C}$ is homotopical and Reedy cofibrant if and only if for all $x \in K_{m}$ the induced diagram $x^{*} X$ is homotopical and Reedy cofibrant. The cofibrancy statement follows by Lemma 1.10 .

It is clear that if $X$ is homotopical then so are all $x^{*} X$. In order to prove the converse it suffices to consider the generating weak equivalences of $D K$. Let $x \in K_{m}$, $y \in K_{n}$ and $\varphi:[m] \hookrightarrow[n]$ be such that $x=y \varphi$ and $y \nu$ is a degenerate edge where $v:[1] \rightarrow[n]$ is defined by $v(0)=\varphi(m)$ and $v(1)=n$. We need to prove that $X \varphi$ is a weak equivalence in $\mathcal{C}$. First, let's assume that $\varphi(m)=n$, then $\varphi$ is a weak equivalence when seen as a morphism $\varphi \rightarrow \operatorname{id}_{[n]}$ in $D[n]$. Therefore $X \varphi=\left(y^{*} X\right) \varphi$ is a weak equivalence since $y^{*} X$ is a homotopical diagram. Next, assume that $\varphi(m)<n$, then $v$ is injective and can be seen as a morphism $y v \rightarrow y$ in $D K$ and we have a commutative diagram on the left in $\Delta_{\sharp}$ which can be reinterpreted as a diagram in the middle in $D K$ which in turn yields the diagram on the right in $\mathcal{C}$ (here $\varepsilon_{i}:[0] \rightarrow[k]$ is the morphism with image $i$ ).
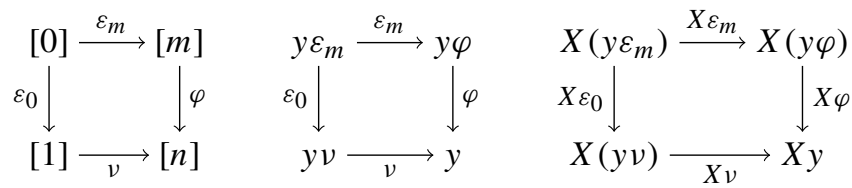

Now, $\varepsilon_{m}$ and $v$ are weak equivalences when seen as morphisms of $D[m]$ and $D[n]$ respectively. Thus $X \varepsilon_{m}$ and $X \nu$ are weak equivalences. The edge $y \nu$ is degenerate, i.e. $y v=y \varepsilon_{n} \sigma_{0}$, so the diagram $(y v)^{*} X: D[1] \rightarrow \mathcal{C}$ factors through $\left(y \varepsilon_{n}\right)^{*} X: D[0] \rightarrow$ $\mathcal{C}$. Since all morphisms of $D[0]$ are weak equivalences it follows that $(y v)^{*} X$ sends all morphisms, including $\varepsilon_{0}$ above, to weak equivalences thus $X \varepsilon_{0}$ is a weak equivalence and hence so is $X \varphi$.

\section{Reedy lifting properties}

In Sect. 1 we introduced Reedy lifting properties. Let $f: I \rightarrow J$ be a homotopical functor of homotopical direct categories and $F: \mathcal{C} \rightarrow \mathcal{D}$ an exact functor of cofibration categories. We say that $f$ has the Reedy left lifting property with respect to $F$ (or $F$ has the Reedy right lifting property with respect to $f$ ) if every lifting problem 


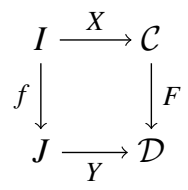

where $X$ and $Y$ are homotopical Reedy cofibrant diagrams has a solution that is also a homotopical Reedy cofibrant diagram.

The previous proposition immediately implies the following.

Corollary 3.1 Let $i: K \rightarrow L$ be a simplicial map and $F: \mathcal{C} \rightarrow \mathcal{D}$ an exact functor between cofibration categories. Then $\mathrm{N}_{\mathrm{f}} F$ has the right lifting property with respect to $i$ if and only if $F$ has the Reedy right lifting property with respect to Di.

Our present goal is to verify that $\mathrm{N}_{\mathrm{f}} \mathcal{C}$ is a quasicategory, i.e. that it has the right lifting property with respect to the inner horn inclusions, that is inclusions $\Lambda^{i}[m] \hookrightarrow \Delta[m]$ for $0<i<m$. To this end we employ the results of Sect. 1 and verify the corresponding Reedy lifting properties of the sieves $D \Lambda^{i}[m] \hookrightarrow D[m]$ induced by the inner horn inclusions. We will proceed by comparing both $D[m]$ and $D \Lambda^{i}[m]$ to $[m]$ and various "generalized inner horns".

Lemma 3.2 For every $m \geq 0$ the functor $p_{[m]}: D[m] \rightarrow[m]$ is a homotopy equivalence of homotopical categories.

Proof Let $f:[m] \rightarrow D[m]$ be the functor that sends $i \in[m]$ to the standard inclusion $[i] \hookrightarrow[m]$. This is a homotopical functor and we have $p_{[m]} f=\operatorname{id}_{[m]}$. We will verify that $f p_{[m]}$ is weakly equivalent to $\operatorname{id}_{D[m]}$ which will finish the proof.

To this end define $s: D[m] \rightarrow D[m]$ as follows. Represent an object $x \in D[m]$ as a non-empty finite non-decreasing sequence of elements of $[m]$. Then $s(x)$ is obtained by inserting one extra occurrence of each of the elements $0,1, \ldots, p_{[m]}(x)$ into $x$. Every such element $i$ is added "at the end" of the (possibly empty) block of $i$ s already present in $x$. This explains the functoriality of $s$. Namely, given $\varphi: x \rightarrow y$ and $i \leq p_{[m]}(x)$, the map $s(\varphi)$ acts on the "old" occurrences of $i$ as $\varphi$ does and sends the "new" occurrences to the "new" occurrences. Thus the functor $s$ is homotopical and admits natural weak equivalences

$$
\mathrm{id} \stackrel{\sim}{\longrightarrow} s \stackrel{\sim}{\longleftarrow} f p_{[m]}
$$

where the map on the left inserts $x$ onto the "old" occurrences in $s(x)$ and the right one inserts $f p_{[m]}(x)$ onto the "new" ones.

Let $A \subseteq[m]$, we define the generalized horn $\Lambda^{A}[m]$ as the simplicial subset of $\Delta[m]$ generated by its codimension 1 faces containing all vertices of $A$ (equivalently, codimension 1 faces lying opposite of vertices not in $A$ ). Observe that $\Lambda^{\{i\}}[m]=$ $\Lambda^{i}[m]$.

Lemma 3.3 The inclusion functor $D \Lambda^{\{1, \ldots, m-1\}}[m] \hookrightarrow D[m]$ induces a weak equivalence $\mathcal{C}_{\mathrm{R}}^{D[m]} \rightarrow \mathcal{C}_{\mathrm{R}}^{D \Lambda^{\{1, \ldots, m-1\}}[m]}$ for every cofibration category $\mathcal{C}$ and each $m \geq 2$. 
Proof The functor $\mathcal{C}_{\mathrm{R}}^{D[m]} \rightarrow \mathcal{C}_{\mathrm{R}}^{D \Lambda^{\{1, \ldots, m-1\}}[m]}$ is exact by Lemma 1.10. By Proposition 1.7 (3) it suffices to verify the statement for the levelwise structures and hence it will be enough to show that the composite $D \Lambda^{\{1, \ldots, m-1\}}[m] \hookrightarrow D[m] \rightarrow[m]$ induces a weak equivalence with respect to the levelwise structures.

In the diagram

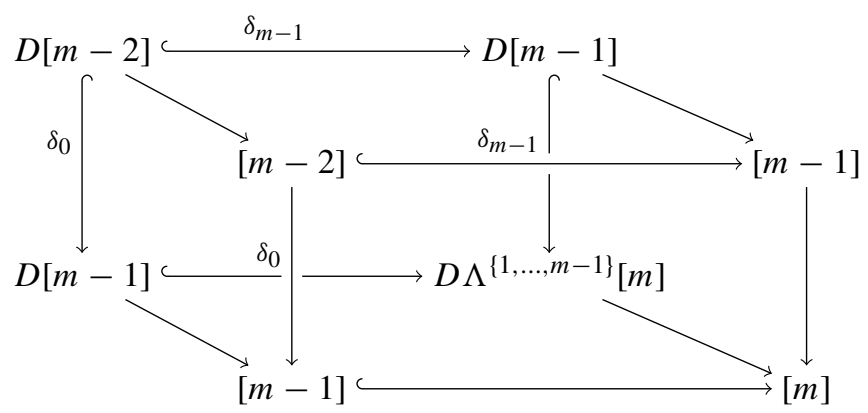

the back square is a pushout of two sieves hence it induces a homotopy pullback of the associated categories of Reedy cofibrant diagrams by Lemma 1.12. The front square is a pushout along a sieve, but the vertical map is not a sieve. Nonetheless, the conclusion of Lemma 1.12 holds because of a particularly simple form of the latching categories in totally ordered sets so that a map of diagrams $[m-1] \rightarrow \mathcal{C}$ is a Reedy cofibration if and only if it is one when restricted along both $\delta_{0}$ and $\delta_{m-1}$. Hence both squares induce homotopy pullbacks on levelwise categories of diagrams and then the assumptions of the Gluing Lemma are satisfied by Lemma 3.2 which finishes the proof.

An interval is a subset of $[m]$ of the form $\{x \in[m] \mid i \leq x \leq j\}$ for some $i \leq j \in$ [m]. In the next lemma we will consider generalized horns $\Lambda^{A}[m]$ with $A \subseteq[m]$ such that $[m] \backslash A$ is not an interval (e.g. $A=\{1, \ldots, m-1\}$ ). Such horns are called generalized inner horns.

Lemma 3.4 Let $A \subseteq B$ be subsets of $[m]$ whose complements are not intervals. Then the inclusion $\Lambda^{B}[m] \hookrightarrow \Lambda^{A}[m]$ is a composite of pushouts of inner horn inclusions in dimensions at most $m-|A|$. Moreover, all these horns are attached along injective maps.

Proof This follows by the proof of [10, Proposition 2.12 (iv)].

It will now follow that $\mathrm{N}_{\mathrm{f}} \mathcal{C}$ takes values in quasicategories. In fact, we show more. Recall that an inner fibration is a simplicial map with the right lifting property with respect to the inner horn inclusions.

Proposition 3.5 The functor $\mathrm{N}_{\mathrm{f}}$ carries fibrations of cofibration categories to inner fibrations. In particular, if $\mathcal{C}$ is a cofibration category, then $\mathrm{N}_{\mathrm{f}} \mathcal{C}$ is a quasicategory.

Proof By Lemma 1.11 it suffices to check that $D \Lambda^{i}[m] \hookrightarrow D[m]$ induces a weak equivalence $\mathcal{C}_{\mathrm{R}}^{D[m]} \rightarrow \mathcal{C}_{\mathrm{R}}^{D \Lambda^{i}[m]}$ for every cofibration category $\mathcal{C}$ and $0<i<m$. By Lemma 3.3 it will be enough to check that $D \Lambda^{\{1, \ldots, m-1\}}[m] \hookrightarrow D \Lambda^{i}[m]$ induces 
a weak equivalence $\mathcal{C}_{\mathrm{R}}^{D \Lambda^{i}[m]} \rightarrow \mathcal{C}_{\mathrm{R}}^{D \Lambda^{\{1, \ldots, m\}}[m]}$ for every cofibration category $\mathcal{C}$ and $0<i<m$.

That follows by an induction with respect to $m$ since this inclusion is built out of pushouts of horn inclusions in dimensions below $m$ by Lemma 3.4. Since these are pushouts along injective maps Lemma 1.12 says that they induce pullbacks of cofibration categories of Reedy diagrams.

In the remainder of this section we will verify some auxiliary lifting properties. First, we consider Reedy lifting properties of [0] $\hookrightarrow D E[1]$ which will be dealt with by constructing an explicit contraction of $D E[1]=D E(1)$ (here, $E(1)$ stands for the groupoid freely generated by one morphism $0 \rightarrow 1$ and $E[1]$ for its nerve).

Lemma 3.6 The functor $f:[0] \rightarrow D E(1)$ given by the sequence $0 \in D E(1)$ is a homotopy equivalence of homotopical categories.

Proof The proof is similar to that of Lemma 3.2. This time objects of $D E(1)$ are represented as arbitrary finite non-empty binary sequences. Let $p: D E(1) \rightarrow[0]$ be the unique functor to [0] and let $s: D E(1) \rightarrow D E(1)$ append a new 0 to every sequence (as before, $s(\varphi)$ acts on "old" elements as $\varphi$ and sends the "new" 0 to the "new" 0$)$. Every morphism of $E(1)$ is an isomorphism so the homotopical structure on $D E(1)$ is the maximal one. Hence the functor $s$ is homotopical and admits natural weak equivalences

$$
\mathrm{id} \stackrel{\sim}{\longrightarrow} s \stackrel{\sim}{\longleftarrow} f p
$$

where the map on the left inserts $x$ onto the "old" occurrences in $s(x)$ and the right one inserts $f p(x)$ onto the "new" 0 .

Before completing the main result of this section we record a corollary which considerably simplifies constructions of $E$ [1]-homotopies. An equivalence in a quasicategory $\mathcal{C}$ is a morphism classified by a map $\Delta[1] \rightarrow \mathcal{C}$ that extends along $\Delta[1] \hookrightarrow E[1]$. By [10, Proposition 4.22] a morphism is a equivalence in $\mathcal{C}$ if and only if it becomes an isomorphism in its homotopy category.

Corollary 3.7 For a cofibration category $\mathcal{C}$ a homotopical Reedy cofibrant diagram $X: D[1] \rightarrow \mathcal{C}$ is an equivalence when seen as a morphism of $\mathrm{N}_{\mathrm{f}} \mathcal{C}$ if and only if it is homotopical with respect to $D \widehat{[1]}$.

Proof If $X$ is an equivalence, then it extends to $D E[1]$. Hence it is homotopical with respect to $D \widehat{[1]}$.

Conversely, consider a diagram

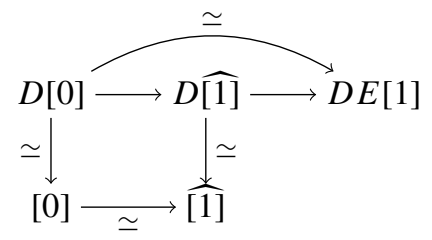

where the indicated maps are homotopy equivalences, the vertical ones by (the proof of) Lemma 3.2, the top one by Lemma 3.6 and the bottom one by direct inspection. 
Hence so is the map $D \widehat{[1]} \rightarrow D E[1]$ which is also a sieve so that the induced restriction functor $\mathcal{C}_{\mathrm{R}}^{D E[1]} \rightarrow \mathcal{C}_{\mathrm{R}}^{D[1]}$ is an acyclic fibration and thus every homotopical Reedy cofibrant diagram on $D \widehat{D[1]}$ extends to one on $D E[1]$.

An isofibration is a simplicial map between quasicategories with the right lifting property with respect to the inclusion $\Delta[0] \rightarrow E[1]$.

Proposition 3.8 The functor $\mathrm{N}_{\mathrm{f}}$ carries fibrations of cofibration categories to isofibrations.

Proof By Lemma 1.11 it suffices to check that $D[0] \hookrightarrow E(1)$ induces a weak equivalence $\mathcal{C}_{\mathrm{R}}^{D E(1)} \rightarrow \mathcal{C}_{\mathrm{R}}^{D[0]}$ for every cofibration category $\mathcal{C}$. Lemma 3.6 asserts that this is the case for the composite

$$
[0] \longleftrightarrow D[0] \longleftrightarrow D E(1)
$$

while Lemma 3.2 says the same for the first functor. Thus the conclusion follows by 2-out-of-3.

Proposition 3.9 The functor $\mathrm{N}_{\mathrm{f}}$ carries acyclic fibrations of cofibration categories to acyclic Kan fibrations.

Proof This follows from Lemmas 1.11 and 1.14 and the fact that $D \partial \Delta[m] \hookrightarrow D[m]$ is a sieve for all $m$.

Before we are able to formulate the final results of this section we need to introduce marked simplicial complexes.

Definition 3.10 A marked simplicial complex is a simplicial set $K$ equipped with an embedding $K \hookrightarrow N P$ where $P$ is a homotopical poset.

Marked simplicial complexes can be seen as certain special marked simplicial sets which are sometimes used to provide some extra flexibility to the theory of quasicategories.

We extend the definition of $D K$ to a marked simplicial complex $K$ as follows. The underlying category of $D K$ is the same as previously, but the homotopical structure is created by the inclusion $D K \hookrightarrow D P$. This agrees with the old definition when $P$ has the trivial homotopical structure.

Moreover, for a marked simplicial complex $K$ we define a homotopical poset $\mathrm{Sd} K$ as the full subcategory of $D K$ spanned by the non-degenerate simplices of $K$ and with the homotopical structure inherited from $D P$. The category $\operatorname{Sd} K$ is known as the barycentric subdivision of $K$ hence the notation (by analogy we may think of $D K$ as the fat barycentric subdivision of $K$ ). It is indeed a poset since its objects can be identified with finite non-empty totally ordered subsets of $P$ that correspond to non-degenerate simplices of $K$ (just as in the classical definition of an ordered simplicial complex above) and morphisms with inclusions of such subsets. With this interpretation an inclusion $A \subseteq B$ is a weak equivalence if and only if $\max A \rightarrow \max B$ is a weak equivalence of $P$ (of course, if $P$ has the trivial homotopical structure, then 
this condition reduces to $\max A=\max B$ ). In the case when $K=N P$ we will usually write $\mathrm{Sd} P$ in place of $\mathrm{Sd} K$.

The next two lemmas will allow us to reduce constructions of diagrams over $D K$ to constructions of diagrams over $\mathrm{Sd} K$.

Lemma 3.11 For any marked simplicial complex $K$ the inclusion $f: \operatorname{Sd} K \rightarrow D K$ is a homotopy equivalence.

Proof The construction is a minor modification of the one used in Lemma 3.2. Let $P$ denote the underlying homotopical poset of $K$. We define $q_{K}: D K \rightarrow \operatorname{Sd} K$ by sending each simplex of $K$ seen as a map $[k] \rightarrow P$ to its image and $s: D K \rightarrow D K$ by inserting one extra occurrence of each $p \in P$ that is already present in a given $x \in D K$. Just as in Lemma 3.2 a new occurrence is inserted at the end of the block of the old occurrences which yields analogous weak equivalences

$$
\mathrm{id} \stackrel{\sim}{\longrightarrow} s \stackrel{\sim}{\longleftarrow} f q_{K} .
$$

Moreover, $q_{K} f=\mathrm{id}_{\mathrm{Sd} K}$ which finishes the proof.

Lemma 3.12 Let $K \hookrightarrow L$ be an injective map of finite marked simplicial complexes (which means that it covers an injective homotopical map of the underlying homotopical posets). Then for every cofibration category $\mathcal{C}$ the inclusion $D K \cup \operatorname{Sd} L \hookrightarrow D L$ induces an acyclic fibration $\mathcal{C}_{\mathrm{R}}^{D L} \rightarrow \mathcal{C}_{\mathrm{R}}^{D K \cup \operatorname{Sd} L}$.

Proof We have the following pushout square of sieves between homotopical direct categories on the left and hence a pullback square of cofibration categories on the right by Lemma 1.12 .
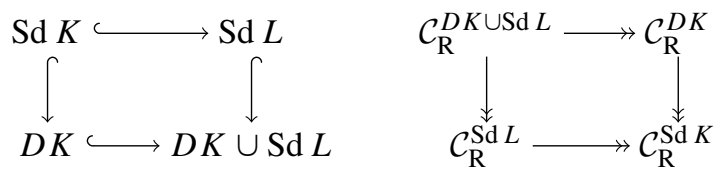

The fibration $\mathcal{C}_{\mathrm{R}}^{D K} \rightarrow \mathcal{C}_{\mathrm{R}}^{\mathrm{Sd} K}$ is acyclic by Lemma 3.11 and therefore so is $\mathcal{C}_{\mathrm{R}}^{D K \cup S d L} \rightarrow \mathcal{C}_{\mathrm{R}}^{\mathrm{Sd} L}$. Moreover, we have a triangle of fibrations

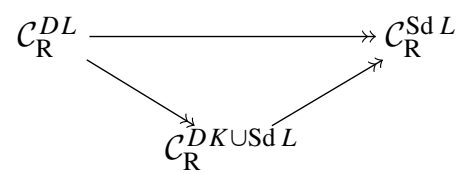

where $\mathcal{C}_{\mathrm{R}}^{D L} \rightarrow \mathcal{C}_{\mathrm{R}}^{\mathrm{Sd} L}$ is acyclic again by Lemma 3.11 and thus so is $\mathcal{C}_{\mathrm{R}}^{D L} \rightarrow \mathcal{C}_{\mathrm{R}}^{D K \cup S d L}$.

For future reference we will reinterpret lifting properties for special outer horns in terms of certain homotopical structures on categories $D \Lambda^{0}[m]$ and $D \Lambda^{m}[m]$ (special outer horns in a quasicategory $\mathcal{C}$ are diagrams $\Lambda^{0}[m] \rightarrow \mathcal{C}$ that carry $0 \rightarrow 1$ to an equivalence and diagrams $\Lambda^{m}[m] \rightarrow \mathcal{C}$ that carry $m-1 \rightarrow m$ to an equivalence).

For each $m>1$ let $\langle m$ ] denote the homotopical poset with the underlying poset $[m]$ and $0 \stackrel{\sim}{\rightarrow} 1$ as the only non-identity weak equivalence. Similarly, let $[m\rangle$ denote 
the homotopical poset with the underlying poset $[m]$ and $m-1 \stackrel{\sim}{\rightarrow} m$ as the only non-identity weak equivalence. Let $\Lambda^{0}\langle m]$ and $\Lambda^{m}[m\rangle$ denote the outer horns seen as marked simplicial complexes with the underlying homotopical posets $\langle m]$ and $[m\rangle$.

Lemma 3.13 For every cofibration category $\mathcal{C}$ the inclusion $D \Lambda^{0}\langle m] \hookrightarrow D\langle m]$ induces a weak equivalence $\mathcal{C}_{\mathrm{R}}^{D\langle m]} \rightarrow \mathcal{C}_{\mathrm{R}}^{D \Lambda^{0}\langle m]}$.

The same holds for $D \Lambda^{m}[m\rangle \hookrightarrow D[m\rangle$.

Proof By Lemma 1.15 it will suffice to see that the inclusion $D \Lambda^{0}\langle m] \hookrightarrow D\langle m]$ has the Reedy left lifting property with respect to all fibrations of cofibration categories.

By Proposition 2.6 every Reedy lifting problem of $D \Lambda^{0}\langle m] \hookrightarrow D\langle m]$ against a fibration of cofibration categories $P: \mathcal{C} \rightarrow \mathcal{D}$ is equivalent to a problem of lifting $\Lambda^{0}\langle m] \hookrightarrow\langle m]$ against $\mathrm{N}_{\mathrm{f}} P$ where the latter is an inner isofibration by Propositions 3.5 and 3.8 and the horn is special by Corollary 3.7. Hence it has a solution by [10, Theorem 4.13].

The same argument works for $D \Lambda^{m}[m\rangle \hookrightarrow D[m\rangle$.

We briefly recall the join of simplicial sets. It will be used in the proof of the next lemma and in the following section. As a functor $\star: \Delta \times \Delta \rightarrow \Delta$ it is defined by concatenation: $[m],[n] \mapsto[m+1+n]$. Then the general join is defined as the unique functor sSet $\times \mathrm{sSet} \rightarrow \mathrm{sSet}$ which agrees with the above on the representable simplicial sets and such that for each $K$ the resulting functor $K \star-$ : sSet $\rightarrow K \downarrow$ sSet preserves colimits. More explicitly, the join of simplicial sets $K$ and $L$ can be described as

$$
(K \star L)_{p}=\coprod_{m+1+n=p} K_{m} \times L_{n}
$$

where $m, n \geq-1$ and $K_{-1}$ and $L_{-1}$ are understood to be one element sets.

The functor $K \star-:$ sSet $\rightarrow K \downarrow$ sSet has a right adjoint. Its value at an object $X: K \rightarrow L$ is called the slice of $X$ under $L$ and denoted by $X \backslash L$. See [9, Section 3] for details.

Let $[k+\widetilde{1}+m]$ denote a homotopical category with underlying category $[k+1+m]$ and $k \stackrel{\sim}{\rightarrow} k+1$ as the only non-identity weak equivalence. Let $\Lambda^{[k]}[k+\widetilde{1}+m]$ denote the generalized horn $\Lambda^{[k]}[k+1+m]$ seen as a marked simplicial complex with the underlying homotopical poset $[k+\widetilde{1}+m]$. The next lemma is a generalization of the previous one.

Lemma 3.14 The inclusion $D \Lambda^{[k]}[k+\widetilde{1}+m] \hookrightarrow D[k+\widetilde{1}+m]$ has the Reedy left lifting property with respect to all fibrations of cofibration categories. Hence for any cofibration category $\mathcal{C}$ it induces a weak equivalence $\mathcal{C}_{\mathrm{R}}^{D[k+\widetilde{1}+m]} \rightarrow \mathcal{C}_{\mathrm{R}}^{D \Lambda^{[k]}[k+\widetilde{1}+m]}$.

Proof The case of $k=0$ is just the previous lemma (with $m$ replaced by $1+m$ ). The case of $k>0$ can be reduced to the case of $k=0$ as follows. We have $[k+1+m] \cong$ $[k] \star[m]$ and $\Lambda^{[k]}[k+1+m] \cong \Delta[k] \star \partial \Delta[m]$ and hence it will suffice to solve every lifting problem 


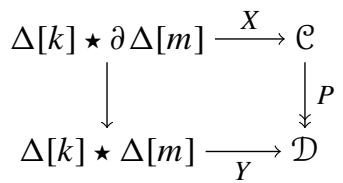

where $X$ and $Y$ send the edge $k \rightarrow k+1$ to an equivalence and $P$ is an inner isofibration (by Proposition 2.6). This problem is equivalent to

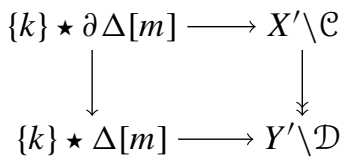

where $X^{\prime}$ and $Y^{\prime}$ are the restrictions of $X$ and $Y$ to $\Delta[k-1]$ so that the resulting horn is special (under identifications $\{k\} \star \Delta[m] \cong \Delta[1+m]$ and $\{k\} \star \partial \Delta[m] \cong \Lambda^{0}[1+m]$ ). The map $X^{\prime} \backslash \mathcal{C} \rightarrow Y^{\prime} \backslash \mathcal{D}$ is an inner isofibration between quasicategories by [10, Theorem 3.19]. Thus a solution exists by the case of $k=0$.

We conclude this section with a technical observation about limits of cofibration categories. Corollary 3.16.1 below is a version of Lemma 1.12.

Lemma 3.15 Let $f: K \rightarrow L$ be a surjective simplicial map. Then for every cofibration category $\mathcal{C}$, a diagram $X: D L \rightarrow \mathcal{C}$ is Reedy cofibrant if and only if $f^{*} X$ is.

Proof For a simplex $y \in L_{m}$ pick a simplex $x \in K_{m}$ such that $f x=y$. Then the induced functor of latching categories $\partial(D K \downarrow x) \rightarrow \partial(D L \downarrow y)$ is an isomorphism since both are essentially copies of $\partial\left(\Delta_{\sharp \downarrow}[\mathrm{m}]\right)$. Thus the latching map of $K$ at $x$ is a cofibration if and only if the latching map of $L$ at $y$ is.

Corollary 3.16 (1) If

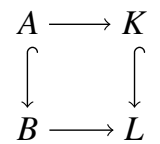

is a pushout square of simplicial sets (along a monomorphism $A \hookrightarrow B$ ), then the square of cofibration categories<smiles>[R2][R2]#[R10][R2]#[R]</smiles>

is a pullback.

(2) If a simplicial set $K$ is a colimit of a sequence of monomorphisms

$$
K_{0} \hookrightarrow K_{1} \hookrightarrow K_{2} \hookrightarrow \cdots,
$$


then $\mathcal{C}_{\mathrm{R}}^{D K}$ is the limit of the tower of fibrations

$$
\cdots \rightarrow \mathcal{C}_{\mathrm{R}}^{D K_{2}} \rightarrow \mathcal{C}_{\mathrm{R}}^{D K_{1}} \rightarrow \mathcal{C}_{\mathrm{R}}^{D K_{0}}
$$

Proof We verify the first statement, the proof of the second one is similar.

By Lemma 2.5 , it is enough to verify that a diagram $X: D L \rightarrow \mathcal{C}$ is homotopical Reedy cofibrant if and only if its restrictions to $D B$ and $D K$ are. For cofibrancy, it follows by Lemma 3.15 applied to the surjection $B \amalg K \rightarrow L$.

For the other part, assume that $X \mid D B$ and $X \mid D K$ are homotopical. Take $y \in L_{n}$ and $\varphi:[m] \hookrightarrow[n]$ such that $y \varphi \rightarrow y$ is a generating weak equivalence in $D L$ (i.e. $y v$ is a degenerate edge of $L$ where $v:[1] \rightarrow[n]$ is defined by $v(0)=\varphi(m)$ and $v(1)=n)$. It will be enough to verify that it arises from a weak equivalence in $D B$ or $D K$. By 2-out-of-6 we can assume that $m=0, n=1, \varphi=\delta_{1}$ and $v=\mathrm{id}_{[1]}$ so that $y v=y$ (if $v$ is not injective, the conclusion is immediate). We consider two cases.

(1) If $y$ is a simplex of $K$, it is also degenerate in $K$ since $K \rightarrow L$ is injective. Thus $y \varphi \rightarrow y$ is a weak equivalence in $D K$.

(2) If $y$ is not a simplex of $K$, then it comes from a unique simplex $x$ of $B$. This simplex is also degenerate since otherwise it would have more distinct degeneracies than $y$ and the square would not be a pushout. In this case, $x \varphi \rightarrow x$ is a weak equivalence in $D B$.

\section{Cocompleteness: the infinite case}

In the last two sections we will verify that $\mathrm{N}_{\mathrm{f}}$ takes values in $\kappa$-cocomplete quasicategories and $\kappa$-cocontinuous functors. From this point on the cases of finitely cocomplete cofibration categories and $\kappa$-cocomplete cofibration categories for $\kappa>\aleph_{0}$ will diverge. The general approaches to both cases are still analogous, but they differ in technical details and there seems to be no way of presenting them in a completely uniform manner. The presence of infinite homotopy colimits allows us to use simpler constructions so we will consider the case of $\kappa>\aleph_{0}$ first (so in this section, $\mathcal{C}$ will denote a $\kappa$-cocomplete cofibration category with $\kappa>\aleph_{0}$, see Sect. 1 ). The remaining case of $\kappa=\aleph_{0}$ will be covered in the next section.

First, we briefly review colimits in quasicategories. Given a simplicial set $K$, we denote $K \star \Delta[0]$ by $K^{\triangleright}$ and call it the cone under $K$. Given a quasicategory $\mathcal{C}$ and a diagram $X: K \rightarrow \mathcal{C}$, any extension of $X$ to $K^{\triangleright}$ is called a cone under $X$. Such a cone $S$ is universal or a colimit of $X$ if for any $m>0$ and any diagram of solid arrows

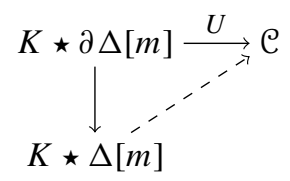

where $U \mid K^{\triangleright}=S$ there exists a dashed arrow making the diagram commute. Given a regular cardinal $\kappa$, we say that $\mathcal{C}$ is $\kappa$-cocomplete all diagrams in $\mathcal{C}$ indexed over $\kappa$-small simplicial sets admit colimits. 
Before we show that $\mathrm{N}_{\mathrm{f}} \mathcal{C}$ is such a quasicategory, we need a few preliminary lemmas. If $I$ is a discrete category, then colimits over [0] $I$ are called wide pushouts. A wide pushout of a diagram $X:[0] \star I \rightarrow \mathcal{C}$ will be denoted by

$$
\coprod_{i \in I} X_{X_{0}}
$$

In order to understand colimits in $\mathrm{N}_{\mathrm{f}} \mathcal{C}$, we need to analyze diagrams over direct categories of the form $D(K \star \Delta[m])$

Lemma 4.1 Let $\mathcal{C}$ be a $\kappa$-cocomplete cofibration category and $K$ a $\kappa$-small simplicial set. If $X: D(K \star \Delta[m]) \rightarrow \mathcal{C}$ is a homotopical Reedy cofibrant diagram, then the induced morphism

$$
X_{[m]} \rightarrow \operatorname{colim}_{D(K \star \Delta[m])} X
$$

is a weak equivalence.

Proof The morphism in question factors as

$$
X_{[m]} \rightarrow \operatorname{colim}_{D[m]} X \rightarrow \operatorname{colim}_{D(K \star \Delta[m])} X
$$

where the first morphism is a weak equivalence by [13, Lemma 3.17] and Lemma 3.2. Thus it will be enough to check that the second one is.

It will suffice to verify that this statement holds when $K$ is a simplex and that it is preserved under coproducts, pushouts along monomorphisms and colimits of sequences of monomorphisms.

Let $K=\Delta[k]$ and let $\iota$ be the composite $[m] \hookrightarrow[k] \star[m] \cong[k+1+m]$. Then we have a commutative square

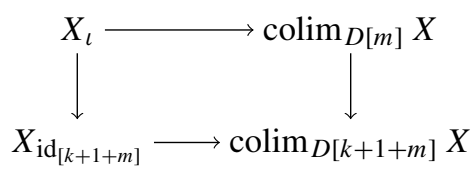

where the left morphism is a weak equivalence since $X$ is homotopical and so are the horizontal ones by the argument above. Thus the right morphism is also a weak equivalence.

Next, consider a pushout square

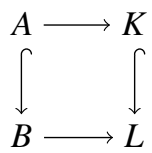

such that the statement holds for $A, B$ and $K$. The functor $-\star \Delta[m]$ preserves pushouts and so does $D$ by Lemma 2.5. Thus in the cube 


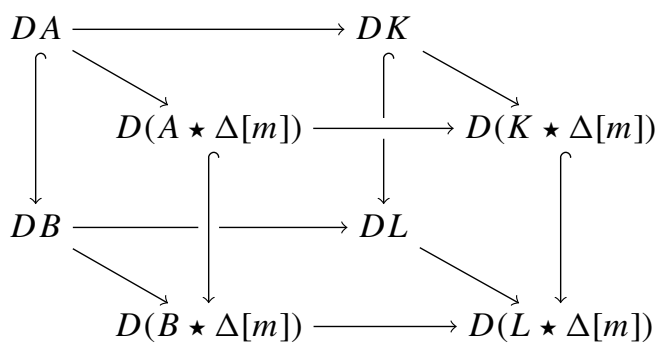

both the front and the back faces are pushouts along sieves and the conclusion follows by [14, Theorem 9.4.1 (1a)] and the Gluing Lemma (here, we also use the fact that a colimit of a diagram whose indexing category is a colimit of a diagram of categories can be computed as an iterated colimit).

The case of colimits of sequences of monomorphisms is similar and we omit it.

The case of coproducts is also similar, but there is a difference in the fact that $-\star \Delta[m]$ does not preserve coproducts. Instead, it sends coproducts to wide pushouts under $\Delta[m]$. Thus if we have a $\kappa$-small family $\left\{K_{i} \mid i \in I\right\}$ of $\kappa$-small simplicial sets and a diagram $X: D\left(\left(\coprod_{i} K_{i}\right) \star \Delta[m]\right) \rightarrow \mathcal{C}$, then there is a canonical isomorphism

$$
\coprod_{i \in I} \operatorname{colim}_{D[m]} X\left(\operatorname{colim}_{D\left(K_{i} \star \Delta[m]\right)} X\right) \cong \operatorname{colim}_{D\left(\left(\coprod_{i \in I} K_{i}\right) \star \Delta[m]\right)} X .
$$

The conclusion follows by the fact that in a cofibration category all the structure morphisms of a wide pushout of acyclic cofibrations are weak equivalences (by [13, Lemma 3.17] since $\widehat{[0] \star I}$ is contractible to its cone object as a homotopical category).

Note that for any simplicial set $K$ there is a unique functor $p_{K}: D\left(K^{\triangleright}\right) \rightarrow(D K)^{\triangleright}$ that restricts to the identity of $D K$ and sends all the objects not in $D K$ to the cone point of $(D K)^{\triangleright}$. This functor is homotopical. In the next lemma we use it to compare colimits over $D K$ and $D\left(K^{\triangleright)}\right.$.

Lemma 4.2 Let $\mathcal{C}$ be a $\kappa$-cocomplete cofibration category, $K$ a $\kappa$-small simplicial set and $X: D K \rightarrow \mathcal{C}$ a homotopical Reedy cofibrant diagram. Consider a morphism $f: \operatorname{colim}_{D K} X \rightarrow Y$ and the corresponding cone $\widetilde{T}:(D K)^{\triangleright} \rightarrow \mathcal{C}$. If $T$ is any Reedy cofibrant replacement of $p_{K}^{*} \widetilde{T}$ relative to $D K$ (which exists by Lemma 1.9 ), then $f$ factors as

$$
\operatorname{colim}_{D K} X \rightarrow \operatorname{colim}_{D\left(K^{\triangleright}\right)} T \stackrel{\sim}{\rightarrow} Y .
$$

Proof To verify that the above composite agrees with $f$ it suffices to check that it agrees upon precomposition with $X_{x} \rightarrow \operatorname{colim}_{D K} X$ for all $x \in D K$. That's indeed the case since $T \mid D K=X$.

It remains to check that the latter morphism is a weak equivalence. In the diagram 


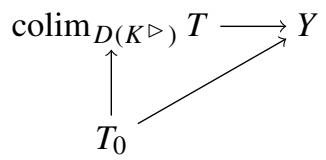

the left morphism is a weak equivalence by Lemma 4.1 and so is the diagonal one since $T$ is a cofibrant replacement of $p_{K}^{*} \widetilde{T}$. Therefore the top morphism is also a weak equivalence.

We will need an augmented version of the $D$ construction. In fact, we will only need to apply it to $[m]$ and $\partial \Delta[m]$ so we define it only in these cases.

We will denote by $D_{\mathrm{a}}[\mathrm{m}]$ the category of all order preserving maps $[\mathrm{k}] \rightarrow[\mathrm{m}]$ including the one with $[k]=[-1]=\varnothing$. A morphism from $x:[k] \rightarrow[m]$ to $y:[l] \rightarrow$ $[m]$ is an injective order preserving map $\varphi:[k] \hookrightarrow[l]$ such that $x=y \varphi$. In other words, $D_{\mathrm{a}}[\mathrm{m}]$ is obtained from $D[\mathrm{~m}]$ by adjoining an initial object. The homotopical structure on $D_{\mathrm{a}}[\mathrm{m}]$ is an extension of the one on $D[\mathrm{~m}]$ where $[-1] \rightarrow[\mathrm{m}]$ is not weakly equivalent to any other object. We will also consider a slightly richer homotopical structure $\widetilde{D}_{\mathrm{a}}[m]$ where $[-1] \rightarrow[m]$ is weakly equivalent to all the constant maps with the value 0 .

The homotopical categories $D_{\mathrm{a}} \partial \Delta[m]$ and $\widetilde{D}_{\mathrm{a}} \partial \Delta[m]$ are the full homotopical subcategories of $D_{\mathrm{a}}[m]$ and $\widetilde{D}_{\mathrm{a}}[\mathrm{m}]$ spanned by the non-surjective maps $[k] \rightarrow[\mathrm{m}]$ [i.e. by the simplices of $\partial \Delta[m]$ including the " $(-1)$-dimensional" one].

Similarly, the homotopical posets $\operatorname{Sd}_{\mathrm{a}}[m], \widetilde{\mathrm{Sd}}_{\mathrm{a}}[m], \mathrm{Sd}_{\mathrm{a}} \partial \Delta[m]$ and $\widetilde{\mathrm{Sd}}_{\mathrm{a}} \partial \Delta[m]$ are the full homotopical subcategories of $D_{\mathrm{a}}[m], \widetilde{D}_{\mathrm{a}}[m], D_{\mathrm{a}} \partial \Delta[m]$ and $\widetilde{D}_{\mathrm{a}} \partial \Delta[m]$ respectively spanned by their objects that are injective as maps $[k] \rightarrow[m]$.

Lemma 4.3 The restriction functors

$$
\begin{array}{rlrl}
\mathcal{C}_{\mathrm{R}}^{D_{\mathrm{a}}[m]} & \rightarrow \mathcal{C}_{\mathrm{R}}^{\mathrm{Sd}_{\mathrm{a}}[m]} & \mathcal{C}_{\mathrm{R}}^{D_{\mathrm{a}} \partial \Delta[m]} & \rightarrow \mathcal{C}_{\mathrm{R}}^{\mathrm{Sd}_{\mathrm{a}} \partial \Delta[m]} \\
\mathcal{C}_{\mathrm{R}}^{\widetilde{\mathrm{D}}_{\mathrm{a}}[m]} & \rightarrow \mathcal{C}_{\mathrm{R}}^{\widetilde{\operatorname{Sd}}_{\mathrm{a}}[m]} & \mathcal{C}_{\mathrm{R}}^{\left.\widetilde{D}_{\mathrm{a}} \partial \Delta m\right]} \rightarrow \mathcal{C}_{\mathrm{R}}^{\widetilde{\mathrm{Sd}_{\mathrm{a}} \partial \Delta[m]}}
\end{array}
$$

are all acyclic fibrations.

Proof All these functors are induced by sieves so they are fibrations. We will construct a homotopy inverse to $f: \widetilde{\operatorname{Sd}}_{\mathrm{a}}[m] \hookrightarrow \widetilde{D}_{\mathrm{a}}[m]$ which will restrict to homotopy inverses of all the other sieves in question. The conclusion will follow by Lemma 1.8 (3). The construction is a minor modification of the one used in Lemma 3.2 (and essentially the same as in Lemma 3.11). Namely, we define $q: \widetilde{D}_{\mathrm{a}}[m] \rightarrow{\widetilde{\mathrm{Sd}_{\mathrm{a}}}}_{\mathrm{a}}[m]$ by sending each $[k] \rightarrow[m]$ to its image and $s: \widetilde{D}_{\mathrm{a}}[m] \rightarrow \widetilde{D}_{\mathrm{a}}[m]$ by inserting one extra occurrence of each $i \in[m]$ that is already present in a given $x \in \widetilde{D}_{\mathrm{a}}[m]$. Just as in Lemma 3.2 a new occurrence is inserted at the end of the block of the old occurrences which yields analogous weak equivalences

$$
\mathrm{id} \stackrel{\sim}{\longrightarrow} s \stackrel{\sim}{\longleftarrow} f q .
$$

Moreover, $q f=\mathrm{id}_{\widetilde{S d}_{\mathrm{a}}[m]}$ which finishes the proof.

Homotopical Reedy cofibrant diagrams on $D_{\mathrm{a}}[1]$ will be used to encode cones on diagrams in $\mathrm{N}_{\mathrm{f}} \mathcal{C}$ and the ones which are homotopical with respect to $\widetilde{D}_{\mathrm{a}}$ [1] will cor- 
respond to the universal cones. The following lemma (and, more directly, Lemma 4.5 below) will translate between the universality of such cones in $\mathrm{N}_{\mathrm{f}} \mathcal{C}$ and strict colimits of the corresponding diagrams in $\mathcal{C}$.

\section{Lemma 4.4 The two functors}

(1) $\mathcal{C}_{\mathrm{R}}^{\widetilde{\mathrm{Sd}_{\mathrm{a}}}[m]} \rightarrow \mathcal{C}_{\mathrm{Sd}_{\mathrm{R}} \partial \Delta[m]}^{\widetilde{\mathrm{R}^{2}}}$ and

(2) $\mathcal{C}_{\mathrm{R}}^{\widetilde{D}_{\mathrm{a}}[m]} \rightarrow \mathcal{C}_{\mathrm{R}}^{\widetilde{D}_{\mathrm{a}} \partial \Delta[m]}$

induced by the inclusion $\partial \Delta[m] \hookrightarrow \Delta[m]$ are acyclic fibrations.

Proof Both inclusions $\widetilde{\mathrm{Sd}}_{\mathrm{a}} \partial \Delta[m] \hookrightarrow \widetilde{\mathrm{Sd}}_{\mathrm{a}}[m]$ and $\widetilde{D}_{\mathrm{a}} \partial \Delta[m] \hookrightarrow \widetilde{D}_{\mathrm{a}}[m]$ are sieves hence by Lemma 1.8 (3) it will be enough to prove that they are homotopy equivalences.

(1) Consider two homotopical functors $i_{0}, i_{1}: \operatorname{Sd}_{\mathrm{a}}[m-1] \rightarrow \widetilde{\operatorname{Sd}}_{\mathrm{a}}[m]$ defined as $i_{0} A=\{k+1 \mid k \in A\}$ and $i_{1} A=i_{0} A \cup\{0\}$ for any $A \subseteq[m-1]$. We have $i_{0} A \subseteq i_{1} A$ and the resulting natural transformation induces an isomorphism of homotopical categories $\operatorname{Sd}_{\mathrm{a}}[m-1] \times \widehat{[1]} \rightarrow{\widetilde{\mathrm{Sd}_{\mathrm{a}}}}_{[}[m]$. It follows that $i_{0}$ is a homotopy equivalence since [0] $\hookrightarrow \widehat{[1]}$ is. This homotopy equivalence also restricts to a homotopy equivalence $\operatorname{Sd}_{\mathrm{a}}[m-1] \hookrightarrow \widetilde{\mathrm{Sd}_{\mathrm{a}}} \partial \Delta[m]$ and thus the conclusion follows by the triangle

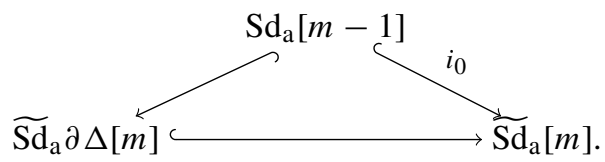

(2) We have a square

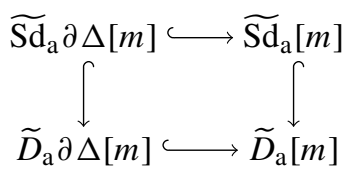

where the top functor is a homotopy equivalence by the first part of the lemma and so are the vertical ones by the proof of Lemma 4.3. Therefore so is the bottom one.

For every $m>0$ each object of $D(K \star \Delta[m])$ can be uniquely written as $x \star \varphi$ with $x \in D_{\mathrm{a}} K$ and $\varphi \in D_{\mathrm{a}}[m]$. This yields a functor $r_{K}: D(K \star \Delta[m]) \rightarrow D_{\mathrm{a}}[m]$ sending $x \star \varphi$ to $\varphi$ to which we associate the left Kan extension

$$
\operatorname{Lan}_{r_{K}}: \mathcal{C}_{\mathrm{R}}^{D(K \star \Delta[m])} \rightarrow \mathcal{C}_{\mathrm{R}}^{D_{\mathrm{a}}[m]}
$$

which can be constructed as

$$
\left(\operatorname{Lan}_{r_{K}} X\right)_{\varphi}=\operatorname{colim}_{D[k]} \varphi^{*} X
$$


where $\varphi:[k] \rightarrow[m]$ (this colimit exists since $\varphi^{*} X$ is Reedy cofibrant by Lemma 2.1). Analogously, we have a functor $s_{K}: D(K \star \partial \Delta[m]) \rightarrow D_{\mathrm{a}} \partial \Delta[m]$ and the associated left Kan extension

$$
\operatorname{Lan}_{S_{K}}: \mathcal{C}_{\mathrm{R}}^{D(K \star \partial \Delta[m])} \rightarrow \mathcal{C}_{\mathrm{R}}^{D_{\mathrm{a}} \partial \Delta[m]}
$$

We form pullbacks (the front and back squares of the cube)

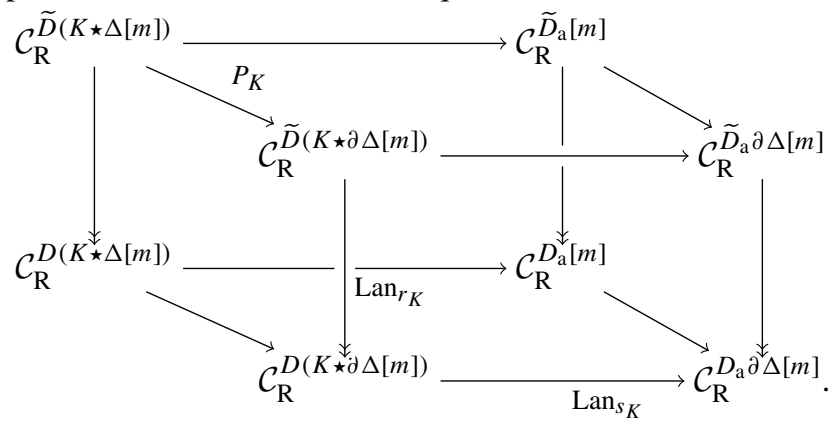

Observe that $\mathcal{C}^{\widetilde{D}(K \star \Delta[m])}$ and $\mathcal{C}^{\widetilde{D}(K \star \partial \Delta[m])}$ are just atomic notations for the pullbacks above, i.e. $\widetilde{D}(K \star \Delta[m])$ and $\widetilde{D}(K \star \partial \Delta[m])$ are $n o t$ homotopical categories for general $K$, although they will be interpreted as such when $K$ is a simplex.

Lemma 4.5 The induced functor $P_{K}: \mathcal{C}_{\mathrm{R}}^{\widetilde{D}(K \star \Delta[m])} \rightarrow \mathcal{C}_{\mathrm{R}}^{\widetilde{D}(K \star \partial \Delta[m])}$ is an acyclic fibration for every $\kappa$-small simplicial set $K$.

Proof First, we verify that $P_{K}$ is a fibration. The categories $\mathcal{C}_{\mathrm{R}}^{\widetilde{D}(K \star \Delta[m])}$ and $\mathcal{C}_{\mathrm{R}}^{\widetilde{D}(K \star \partial \Delta[m])}$ are full subcategories of $\mathcal{C}_{\mathrm{R}}^{D(K \star \Delta[m])}$ and $\mathcal{C}_{\mathrm{R}}^{D(K \star \partial \Delta[m])}$ respectively. They are both closed under taking weakly equivalent objects. Hence $P_{K}$ inherits the desired lifting properties from the fibration $\mathcal{C}_{\mathrm{R}}^{D(K \star \Delta[m])} \rightarrow \mathcal{C}_{\mathrm{R}}^{D(K \star \partial \Delta[m])}$.

For the rest of the argument it will suffice to check that $P_{K}$ is a weak equivalence when $K$ is empty or a simplex and that this property is preserved under coproducts, pushouts along monomorphisms and colimits of sequences of monomorphisms.

When $K$ is empty then the top square of the cube above happens to be a pullback and hence $P_{\varnothing}$ is an acyclic fibration by Lemma 4.4 .

For $K=\Delta[k]$ we will check that $P_{\Delta[k]}$ coincides with

$$
\mathcal{C}_{\mathrm{R}}^{D[k+\widetilde{1}+m]} \rightarrow \mathcal{C}_{\mathrm{R}}^{D \Lambda^{[k]}[k+\widetilde{1}+m]}
$$

and the conclusion will follow from Lemma 3.14. It is enough to verify that a homotopical Reedy cofibrant diagram $X: D[k+1+m] \rightarrow \mathcal{C}$ is homotopical with respect to $D[k+\widetilde{1}+m]$ if and only if the induced morphism

$$
\operatorname{colim}_{D[k]} X \rightarrow \operatorname{colim}_{D[k+1]} X
$$

is a weak equivalence. This follows from Lemma 4.1. The same argument works with $\Lambda^{[k]}[k+1+m]$ in place of $[k+1+m]$, since $\Delta[k+1]$ is contained in $\Lambda^{[k]}[k+1+m]$ for $m>0$. 
If

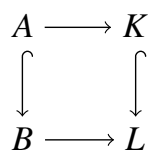

is a pushout square of simplicial sets such that the conclusion holds for $A, B$ and $K$, then there is a pullback square of cofibration categories

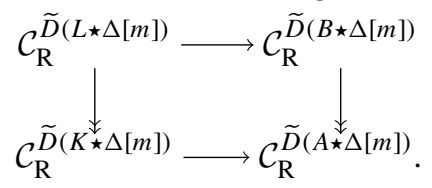

An analogous pullback square with $D$ in the place of $\widetilde{D}$ results directly from Corollary 3.16 (1). This implies the existence of the one above since pullbacks commute with pullbacks. Similarly, there is an analogous pullback square with $\partial \Delta[m]$ in place of $\Delta[m]$. Hence the conclusion for $L$ follows from the Gluing Lemma.

The last two cases depend on the fact that CofCat $_{\kappa}$ is a complete fibration category as follows from [21, Theorem 2.9].

If $K$ is a colimit of a sequence of monomorphisms $K_{0} \hookrightarrow K_{1} \hookrightarrow K_{2} \hookrightarrow \cdots$, then $\mathcal{C}_{\mathrm{R}}^{\widetilde{D}(K \star \Delta[m])}$ is the limit of the tower of fibrations

$$
\cdots \rightarrow \mathcal{C}_{\mathrm{R}}^{\widetilde{D}\left(K_{2} \star \Delta[m]\right)} \rightarrow \mathcal{C}_{\mathrm{R}}^{\widetilde{D}\left(K_{1} \star \Delta[m]\right)} \rightarrow \mathcal{C}_{\mathrm{R}}^{\widetilde{D}\left(K_{0} \star \Delta[m]\right)}
$$

and analogously for $\mathcal{C}_{\mathrm{R}}^{\widetilde{D}(K \star \partial \Delta[m])}$ [this follows from Corollary 3.16 (2), similarly to the previous case]. Therefore, if $P_{K_{i}}$ is a weak equivalence for all $i$, then so is $P_{K}$.

The case of coproducts is handled similarly except that $-\star \Delta[m]$ does not preserve coproducts but carries them to wide pushouts. Hence $\mathcal{C}_{\mathrm{R}}^{\widetilde{D}\left(\left(\amalg_{i} K_{i}\right) \star \Delta[m]\right)}$ is the wide pullback

$$
\prod_{i} \mathcal{C}_{\mathcal{R}^{D m]}} \mathcal{C}_{\mathrm{R}}^{\widetilde{D}\left(K_{i} \star \Delta[m]\right)}
$$

The conclusion follows since the wide pullback functor is an exact functor of fibration categories.

We are ready to characterize colimits in $\mathrm{N}_{\mathrm{f}} \mathcal{C}$ in terms of homotopy colimits in $\mathcal{C}$.

Theorem 4.6 Let $\mathcal{C}$ be a $\kappa$-cocomplete cofibration category, $K$ a $\kappa$-small simplicial set and $S: K^{\triangleright} \rightarrow \mathrm{N}_{\mathrm{f}} \mathcal{C}$. Then $S$ is universal as a cone under $S \mid K$ if and only if the induced morphism

$$
\operatorname{colim}_{D K} S \rightarrow \operatorname{colim}_{D\left(K^{\triangleright}\right)} S
$$

is a weak equivalence (with $S$ seen as a homotopical Reedy cofibrant diagram $D\left(K^{\triangleright}\right) \rightarrow \mathcal{C}$ by Proposition 2.6). Such a cone exists under every diagram $K \rightarrow \mathrm{N}_{\mathrm{f}} \mathcal{C}$. 
Proof If the morphism above is a weak equivalence let $U: K \star \partial \Delta[m] \rightarrow \mathrm{N}_{\mathrm{f}} \mathcal{C}$ extend $S$. The functor $\mathcal{C}_{\mathrm{R}}^{\widetilde{D}(K \star \Delta[m])} \rightarrow \mathcal{C}_{\mathrm{R}}^{\widetilde{D}(K \star \partial \Delta[m])}$ is an acyclic fibration by Lemma 4.5 and thus the corresponding homotopical Reedy cofibrant diagram $\widetilde{D}(K \star \partial \Delta[m]) \rightarrow \mathcal{C}$ prolongs to $\widetilde{D}(K \star \Delta[m]) \rightarrow \mathcal{C}$. Hence $S$ is universal.

Conversely, let $S$ be universal. Define $T: D\left(K^{\triangleright}\right) \rightarrow \mathcal{C}$ as in Lemma 4.2 where we take $f$ to be the identity of $\operatorname{colim}_{D K} S$. Then the induced morphism $\operatorname{colim}_{D K} T \rightarrow$ colim $_{D\left(K^{\triangleright}\right)} T$ is a weak equivalence and so $T$ is universal by the argument above (which proves the existence statement). Therefore $S$ and $T$ are equivalent and hence there exists a homotopical Reedy cofibrant diagram $W: D(K \star E[1]) \rightarrow \mathcal{C}$ which restricts to $S$ on $D(K \star\{0\})$ and to $T$ on $D(K \star\{1\})$, see [9, Proposition 4.4]. In the diagram

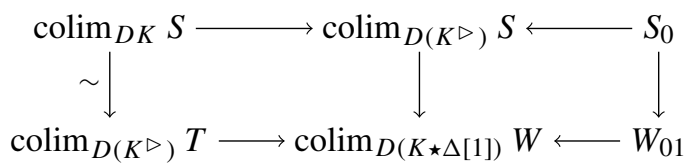

both bottom horizontal morphisms and the top right one are weak equivalences by Lemma 4.1 and so is the right vertical one since the homotopical structure of $D E[1]$ is the maximal one. It follows that $\operatorname{colim}_{D K} S \rightarrow \operatorname{colim}_{D\left(K^{\triangleright}\right)} S$ is also a weak equivalence.

\section{Cocompleteness: the finite case}

In this section we will prove that $\mathrm{N}_{\mathrm{f}} \mathcal{C}$ is finitely cocomplete for any cofibration category. The arguments of the previous section do not directly apply to this case since they heavily use the existence of colimits of Reedy cofibrant diagrams over categories of the form $D K$. Unfortunately, $D K$ is infinite even when $K$ is a finite (non-empty) simplicial set. In order to address this problem, we will filter the category $D K$ by finite subcategories

$$
D^{(0)} K \hookrightarrow D^{(1)} K \hookrightarrow D^{(2)} K \hookrightarrow \cdots
$$

and instead of using a colimit of a Reedy cofibrant diagram $X: D K \rightarrow \mathcal{C}$ we will consider the resulting sequence of finite colimits

$$
\operatorname{colim}_{D^{(0)} K} X \longmapsto \operatorname{colim}_{D^{(1)} K} X \longmapsto \operatorname{colim}_{D^{(2)} K} X \longmapsto \cdots
$$

If $X$ is homotopical this sequence stabilizes in the sense that from some point on (depending on $K$ ) all morphisms are weak equivalences and this stable value is a homotopy colimit of $X$. However, there is no universal bound on when such a sequence stabilizes when $K$ varies and hence we are forced to think of that entire sequence as a homotopy colimit of $X$. It turns out that the proofs of the previous section will work if we carefully substitute such sequences for actual colimits over categories $D K$. The difficult part is constructing such filtrations with all the desired naturality and homotopy invariance which is the main purpose of this section. 
Let $J$ be a homotopical category and $A$ a set of objects of $D J$, we denote the sieve generated by $A$ in $D J$ by $D^{A} J$. Moreover, when $J=[m]$ (possibly with some non-trivial homotopical structure) we will write objects of $D[\mathrm{~m}]$ as non-decreasing sequences of elements of $[\mathrm{m}]$ often using abbreviations like $0^{k} 1^{l}$ to denote the sequence of $k$ os followed by $l 1 \mathrm{~s}$.

The category $D[0]$ can be seen as the category of non-degenerate simplices of a simplicial set $S$ with exactly one non-degenerate simplex in each dimension. As it turns out, the skeleton $\mathrm{Sk}^{k} S$ is weakly contractible for $k$ even but weakly equivalent to the sphere $\Delta[k] / \partial \Delta[k]$ for $k$ odd.

This suggests that the filtration of $D[0]$ by sieves generated by even-dimensional simplices of $S$ should be well-behaved homotopically. We verify that this is the case in the next two lemmas and later generalize it to $D K$ for arbitrary finite simplicial sets $K$.

Lemma 5.1 For each $k$ the functor $t: D^{0^{k} 1 \widehat{[1]}} \rightarrow[0]$ is a homotopy equivalence of homotopical categories.

Proof Represent objects of $D^{0^{k} 1} \widehat{[1]}$ as binary sequences and let $j:[0] \rightarrow D^{0^{k} 1 \widehat{[1]}}$ classify the object 1 . Next, define $s: D^{0^{k} 1} \widehat{[1]} \rightarrow D^{0^{k} 1} \widehat{[1]}$ by appending a trailing 1 to each sequence that doesn't have one. Then there are natural weak equivalences

$$
\operatorname{id}_{D^{0^{k}} \widehat{[1]}} \stackrel{\sim}{\longrightarrow} s \stackrel{\sim}{\longleftarrow} j t .
$$

Moreover, we have $t j=\mathrm{id}_{[0]}$ which finishes the proof.

The images of the composite functors

$$
\operatorname{Sd}[k] \hookrightarrow D[k] \rightarrow D[0] \quad \text { and } \quad \operatorname{Sd} \partial \Delta[k+1] \hookrightarrow D \partial \Delta[k+1] \rightarrow D[0]
$$

are both $D^{0^{k+1}}[0]$. In the next lemma we consider the resulting functors

$$
t: \operatorname{Sd}[k] \rightarrow D^{0^{k+1}}[0] \text { and } t: \operatorname{Sd} \partial \Delta[k+1] \rightarrow D^{0^{k+1}}[0]
$$

Lemma 5.2 Let $k \geq 0$ and let $\mathcal{C}$ be a cofibration category. If $X: D^{0^{k+1}}[0] \rightarrow \mathcal{C}$ is a homotopical Reedy cofibrant diagram, then

(1) the induced morphism

$$
\operatorname{colim}_{\mathrm{Sd} \Delta[k]} t^{*} X \rightarrow \operatorname{colim}_{D^{0^{k+1}}[0]} X
$$

is a weak equivalence when $k$ is even,

(2) the induced morphism

$$
\operatorname{colim}_{S d \partial}{ }_{[k+1]} t^{*} X \rightarrow \operatorname{colim}_{D^{0^{k+1}}[0]} X
$$

is a weak equivalence when $k$ is odd. 
Proof We prove both statements by an alternating induction with respect to $k$.

The functor $\mathrm{Sd}[0] \rightarrow D^{0}[0]$ is an isomorphism, so condition (1) holds for $k=0$.

Next, we assume that condition (2) holds for a given odd $k$ and prove that condition (1) holds for $k+1$. The category $\operatorname{Sd} \partial \Delta[k+1]$ is nothing but the latching category of $D^{0^{k+2}}[0]$ at $0^{k+2}$ and hence the inductive construction of the colimit of $X$ yields a pushout square

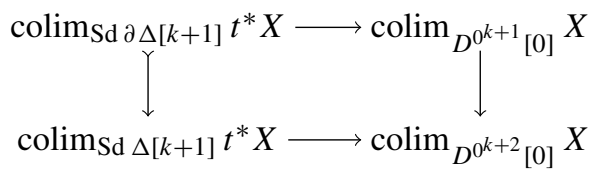

where the top morphism is a weak equivalence by the inductive hypothesis. Since the left vertical morphism is a cofibration, it follows by the Gluing Lemma that the bottom morphism is also a weak equivalence.

Finally, we assume that condition (1) holds for a given even $k$ and prove that condition (2) holds for $k+1$. We have the following diagram of homotopical direct categories

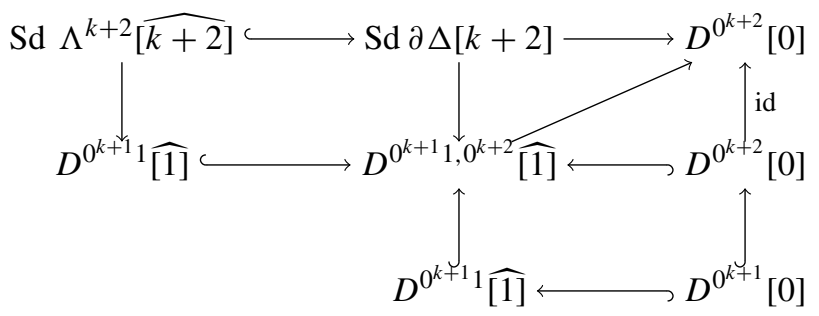

where the indicated maps are sieves, the top left and bottom right squares are pushouts and all functors respect Reedy cofibrant diagrams by Lemma 1.10 (the functor on the very left is induced by $\left.0^{k+2} 1:[k+2] \rightarrow[1]\right)$. Hence there is an induced diagram in $\mathcal{C}$

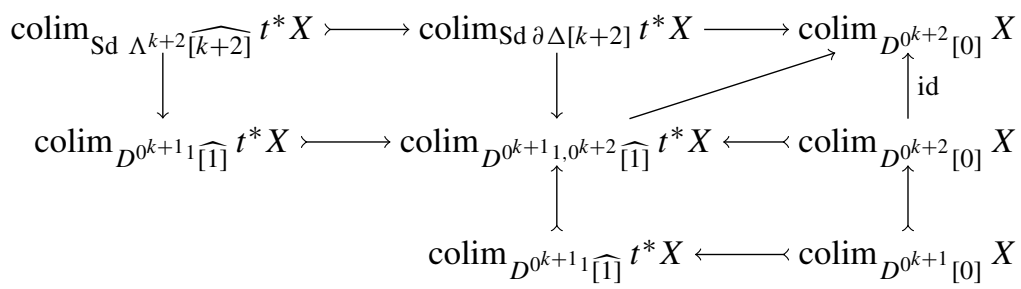

where the indicated maps are cofibrations by [14, Theorem 9.4.1 (1a)] and the top left and bottom right squares are pushouts (here, we use the fact that a colimit of a diagram whose indexing category is a colimit of a diagram of categories can be computed as an iterated colimit).

Thus the proof will be completed when we verify that both morphisms

$$
\begin{gathered}
\operatorname{colim}_{\mathrm{Sd} \Lambda^{\widehat{k+2}}[k \hat{+2]}} t^{*} X \rightarrow \operatorname{colim}_{D^{0^{k+1}} 1 \widehat{[1]}} t^{*} X \\
\operatorname{colim}_{D^{0^{k+1}}[0]} t^{*} X \rightarrow \operatorname{colim}_{D^{0^{k+1}} \widehat{[1]}} X
\end{gathered}
$$


are weak equivalences. For the former we use [13, Lemma 3.17] and Lemmas 5.1 and 3.13. For the latter we use [13, Lemma 3.17], Lemma 5.1 and the inductive assumption.

In the next two lemmas we generalize the filtration of $D[0]$ to $D[m]$ for all $m \geq 0$.

Lemma 5.3 Let $\mathcal{C}$ be a cofibration category. Assume that every fiber of $\varphi:[k] \rightarrow[m]$ has an odd number of elements and let $X: D^{\varphi}[\mathrm{m}] \rightarrow \mathcal{C}$ be a homotopical Reedy cofibrant diagram. Then $X_{\varphi} \rightarrow \operatorname{colim} X$ is a weak equivalence.

Proof We proceed by induction with respect to $m$ (simultaneously for all $\mathcal{C}$ and $X$ ). For $m=0$ the conclusion follows by Lemma 5.2.

If $m>0$, we will prolong $X$ to the augmented sieve $D_{\mathrm{a}}^{\varphi}[m]$ by setting the missing value to an initial object of $\mathcal{C}$ which does not change the colimit. If the fiber of $\varphi$ over $m$ has $k+1$ elements for some even $k$, then $D_{\mathrm{a}}^{\varphi}[m] \cong D_{\mathrm{a}}^{\varphi^{\prime}}[m-1] \times D_{\mathrm{a}}^{0^{k+1}}[0]$ (here, $\varphi^{\prime}$ is the restriction of $\varphi$ to $\left.\varphi^{-1}[m-1]\right)$. We apply Lemma 5.2 in the category $\mathcal{C}_{\mathrm{R}}^{D_{\mathrm{a}}^{\varphi^{\prime}}[m-1]}$ to the corresponding diagram $\widetilde{X}: D_{\mathrm{a}}^{0^{k+1}}[0] \rightarrow \mathcal{C}_{\mathrm{R}}^{D_{\mathrm{a}}^{\varphi^{\prime}}[m-1]}$ (this diagram is indeed Reedy cofibrant by the proof of Lemma 1.13). This way, we obtain a weak equivalence $\widetilde{X}_{k} \rightarrow \operatorname{colim}_{D_{\mathrm{a}}^{0^{k+1}}[0]} \widetilde{X}$ and hence by the inductive assumption the composite

$$
X_{\varphi}=\widetilde{X}_{k, \varphi^{\prime}} \rightarrow \operatorname{colim}_{D_{\mathrm{a}}^{\varphi^{\prime}[m-1]}} \tilde{X}_{k} \rightarrow \operatorname{colim}_{D_{\mathrm{a}}^{\varphi^{\prime}[m-1]}} \operatorname{colim}_{D_{\mathrm{a}}^{0^{k+1}[0]}} \tilde{X} \cong \operatorname{colim} X
$$

is also a weak equivalence.

For each $k, m \geq 0$ we define sets $A_{k, m}$ and $B_{k, m}$ of objects of $D[m]$. We proceed by induction with respect to $m$. First, we set $A_{k, 0}=B_{k, 0}=\{[2 k] \rightarrow[0]\}$. For $m>0$ we set

$$
\begin{aligned}
& B_{k, m}=\{\varphi:[2 k-m] \rightarrow[m] \mid \text { each fiber of } \varphi \text { has an odd number of elements }\} \\
& A_{k, m}=B_{k, m} \cup \bigcup_{i \in[m]} \delta_{i} A_{k, m-1} .
\end{aligned}
$$

We set $D^{(k)}[m]=D^{A_{k, m}}[m]$. In particular, we have $D^{(k)}[0]=D^{[2 k]}[0]$, i.e. $D^{(k)}[m]$ generalizes the filtration of $D[0]$ by sieves corresponding to even-dimensional skeleta of $S$ as discussed on p. 33. There $S$ was defined as a simplicial set with exactly one non-degenerate simplex in each dimension. Similarly, there is a simplicial set $S_{m}$ whose non-degenerate $p$-simplices correspond to all simplicial operators $[p] \rightarrow[m]$ (with degenerate ones adjoined freely). Then the sieve generated by $B_{k, m}$ corresponds to the ( $2 k$ )-skeleton of $S_{m}$. However, these sieves do not match exactly when $m$ varies and the definition of $A_{k, m}$ corrects that.

Lemma 5.4 For every simplicial operator $\chi:[m] \rightarrow[n]$ and $k \geq 0$ we have an inclusion $\chi D^{(k)}[m] \subseteq D^{(k)}[n]$.

Proof It suffices to verify the statement when $\chi$ is an elementary face or degeneracy operator. For the elementary face operators it follows directly from the definition. 
Hence assume that $\chi=\sigma_{j}$ for some $j \in[n]$. We will check that $\sigma_{j} A_{k, n+1} \subseteq D^{(k)}[n]$ by induction with respect to $n$.

If $\varphi:[2 k-n-1] \rightarrow[n+1]$ has all fibers of odd cardinality, then the same holds for $\sigma_{j} \varphi$ except at the fiber over $j$. Then $\sigma_{j} \varphi$ is in the sieve generated by $\varphi^{\prime}:[2 k-n] \rightarrow[n]$ obtained by adding one extra element to the fiber of $\sigma_{j} \varphi$ over $j$ (so that $\varphi^{\prime} \in A_{k, n}$ ).

If $\psi \in A_{k, n}$, then $\sigma_{j} \delta_{i} \psi$ is either equal to $\psi$ or is of the form $\delta_{i^{\prime}} \sigma_{j^{\prime}} \psi$. In the first case the conclusion holds trivially, in the second one it follows by the inductive hypothesis.

Now, we can generalize the filtration of $D[m]$ to $D K$ for arbitrary finite $K$. Let $x \in K_{m}$ and $k \geq 0$. We define a sieve $D^{(k)} K$ in $D K$ as follows. Write $x=x^{\sharp} x^{b}$ with $x^{\sharp}$ non-degenerate and $x^{\mathrm{b}}$ a degeneracy operator (this can be done in exactly one way by the Eilenberg-Zilber Lemma, see e.g. [7, Section II.3]). Define $x$ to be an element of $D^{(k)} K$ if $x^{b} \in D^{(k)}[n]$ (where $n$ is the dimension of $x^{\sharp}$ ). It follows from Lemma 5.4 that this definition coincides with the previous one when $K$ is a simplex.

Lemma 5.5 Every simplicial map $f: K \rightarrow$ L carries $D^{(k)} K$ to $D^{(k)} L$ for all $k \geq 0$.

Proof Let $x \in D^{(k)} K$. Then we have a diagram of simplicial sets

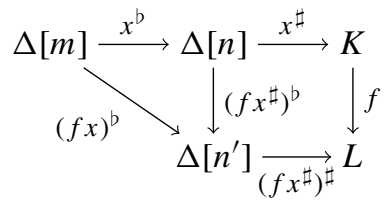

and by definition $x^{b} \in D^{(k)}[n]$. Lemma 5.4 implies that $(f x)^{b} \in D^{(k)}\left[n^{\prime}\right]$ so that $f x \in D^{(k)} L$.

Lemma 5.6 For all $k \geq m$, a cofibration category $\mathcal{C}$ and a homotopical Reedy cofibrant diagram $X: D^{(k)}[m] \rightarrow \mathcal{C}$ the morphism $X_{[m]} \rightarrow \operatorname{colim}_{D^{(k)}[m]} X$ is a weak equivalence.

Proof First, we will check that the morphism $X_{[m]} \rightarrow D^{B_{k, m}}[m]$ is a weak equivalence. Indeed, let $P$ be the subposet of $\mathbb{N}^{m+1}$ consisting of tuples $x=\left(x_{0}, \ldots, x_{m}\right)$ such that each $x_{i}$ is odd and $x_{0}+\cdots+x_{m} \leq 2 k-m+1$. Let $\varphi_{x}$ be the unique object of $D[m]$ whose fiber over each $i \in[m]$ has cardinality $x_{i}$. Then we have $D^{B_{k, m}}[m]=$ $\operatorname{colim}_{x \in P} D^{\varphi_{x}}[\mathrm{~m}]$ since a colimit of a diagram whose indexing category is a colimit of a diagram of categories can be computed as an iterated colimit.

It follows from Lemma 5.3 that for each $x \in P$ the morphism $X_{[m]} \rightarrow$ $\operatorname{colim}_{D^{\varphi_{x}[m]}} X$ is a weak equivalence. The sequence $(1, \ldots, 1)$ is the bottom element of $P$, hence if we consider $P$ as a homotopical poset with all maps as weak equivalences, then $\{(1, \ldots, 1)\} \rightarrow P$ is a homotopy equivalence. It follows by [13, Lemma 3.17] that $X_{[m]} \rightarrow D^{B_{k, m}}[m]$ is a weak equivalence.

We are ready to prove the lemma by induction with respect to $m$. If $m=0$, then the conclusion is a special case of Lemma 5.3. For $m>0$, we consider diagrams $Y^{(k-1)}, Y^{(k)}: \operatorname{Sd} \partial \Delta[m] \rightarrow \mathcal{C}$ defined as

$$
Y_{\varphi}^{(k-1)}=\operatorname{colim}_{D^{\varphi A_{k-1, n}[m]}} X \quad \text { and } \quad Y_{\varphi}^{(k)}=\operatorname{colim}_{D^{\varphi A_{k, n}[m]}} X
$$


where $\varphi:[n] \hookrightarrow[m]$. The resulting morphism $Y^{(k-1)} \rightarrow Y^{(k)}$ is a Reedy cofibration by [14, Theorem 9.4.1 (1a)]. It is also a weak equivalence by the inductive hypothesis

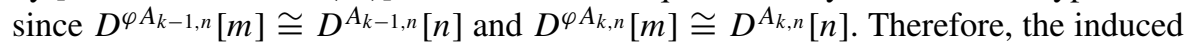
morphism colim $Y^{(k-1)} \rightarrow$ colim $Y^{(k)}$ is an acyclic cofibration. This morphism is also isomorphic to $\operatorname{colim}_{D^{(k-1)} \partial \Delta[m]} X \rightarrow \operatorname{colim}_{D^{(k)} \partial \Delta[m]} X$ since

$$
\begin{aligned}
D^{(k-1)} \partial \Delta[m] & =\operatorname{colim}_{\varphi \in \operatorname{Sd} \partial \Delta[m]} D^{\varphi A_{k-1, n}[m]} \\
\text { and } \quad D^{(k)} \partial \Delta[m] & =\operatorname{colim}_{\varphi \in \operatorname{Sd} \partial \Delta[m]} D^{\varphi A_{k, n}[m] .}
\end{aligned}
$$

Finally, we observe that $D^{B_{k, m}}[m] \cap D^{(k)} \partial \Delta[m]=D^{(k-1)} \partial \Delta[m]$. This yields pushout square

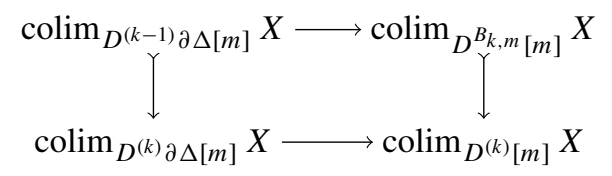

which along with the first part of the proof yields the final conclusion.

Lemma 5.7 For each $k$ the functor $D^{(k)}$ : sSet $\rightarrow$ Cat (i.e. when we disregard the homotopical structures of $\left.D^{(k)} K s\right)$ preserves colimits.

Proof If $K$ is any simplicial set, then $D^{(k)}$ preserves the colimit of its simplices by Lemma 2.5 and the definition of $D^{(k)} K$. Hence for every small category $J$ we have the following sequence of isomorphisms natural in both $K$ and $J$.

$$
\begin{aligned}
\operatorname{Cat}\left(D^{(k)} K, J\right) & \cong \operatorname{Cat}\left(D^{(k)} \operatorname{colim}_{\Delta[m] \rightarrow K} \Delta[m], J\right) \\
& \cong \lim _{\Delta[m] \rightarrow K} \operatorname{Cat}\left(D^{(k)}[m], J\right) \\
& \cong \lim _{\Delta[m] \rightarrow K} \operatorname{sSet}\left(\Delta[m], \operatorname{Cat}\left(D^{(k)}[-], J\right)\right) \\
& \cong \operatorname{sSet}\left(K, \operatorname{Cat}\left(D^{(k)}[-], J\right)\right)
\end{aligned}
$$

It follows that $J \mapsto \operatorname{Cat}\left(D^{(k)}[-], J\right)$ is a right adjoint of $D^{(k)}$ and the conclusion follows.

Finally, we are ready to start translating the results of Sect. 4 to the case of $\kappa=\aleph_{0}$. The following is a counterpart to Lemma 4.1.

Lemma 5.8 Let $\mathcal{C}$ be a cofibration category and $K$ a finite simplicial set. For every homotopical Reedy cofibrant diagram $X: D(K \star \Delta[m]) \rightarrow \mathcal{C}$ and all $k \geq \operatorname{dim} K+$ $1+m$, the induced morphism

$$
X_{[m]} \rightarrow \operatorname{colim}_{D^{(k)}(K \star \Delta[m])} X
$$

is a weak equivalence. 
Proof The proof is analogous to the proof of Lemma 4.1 using Lemma 5.7 in the place of Lemma 2.5 .

For a cofibration category $\mathcal{C}$ we introduce a new cofibration category $\mathcal{C}_{\mathrm{R}}^{\widetilde{\mathbb{N}}}$ (here, $\widetilde{\mathbb{N}}$ does not refer to any homotopical structure on $\mathbb{N}, \mathcal{C}_{\mathrm{R}}^{\widetilde{\mathbb{N}}}$ should be seen as an atomic notation). Its objects are Reedy cofibrant diagrams $X: \mathbb{N} \rightarrow \mathcal{C}$ (i.e. sequences of cofibrations in $\mathcal{C}$ ) that are eventually (homotopically) constant, i.e. such that there is a number $k$ such that for all $l \geq k$ the morphism $X_{k} \rightarrow X_{l}$ is a weak equivalence. A morphism $f: X \rightarrow Y$ of such diagrams is called an eventual weak equivalence if there is $k$ such that for all $l \geq k$ the morphism $f_{l}$ is a weak equivalence in $\mathcal{C}$. This cofibration category is designed as an enlargement of the cofibration category $\mathcal{C}_{\mathrm{R}}^{\widehat{\mathbb{N}}}$ of (homotopically) constant sequences. It is necessary since sequences arising as colimits over filtrations $D^{(-)} K$ are only eventually constant.

Lemma 5.9 If $\mathcal{C}$ is a cofibration category, then the category $\mathcal{C}_{\mathrm{R}}^{\widetilde{\mathbb{N}}}$ with Reedy cofibrations and eventual weak equivalences is also a cofibration category. Moreover, the inclusion $\mathcal{C}_{\mathrm{R}}^{\widehat{\mathbb{N}}} \hookrightarrow \mathcal{C}_{\mathrm{R}}^{\widetilde{\mathbb{N}}}$ is a weak equivalence.

Proof The construction of the cofibration category $\mathcal{C}_{\mathrm{R}}^{\widetilde{\mathbb{N}}}$ is a straightforward modification of the construction of $\mathcal{C}_{\mathrm{R}}^{\mathbb{N}}$, see e.g. [14, Theorem 9.3.5 (1)].

We will verify the approximation properties [21, Proposition 2.2]. By 2-out-of-3 a morphism between homotopically constant sequences is a levelwise weak equivalence if and only if it is an eventual weak equivalence. Hence (App1) holds.

Next, let $X \rightarrow Y$ be a morphism with $X$ homotopically constant and $Y$ eventually constant. Assume that $Y$ is homotopically constant from degree $k$ on. Let $\widetilde{Y}$ be $Y$ shifted down by $k$. Then $\widetilde{Y}$ is homotopically constant and iterated structure morphisms of $Y$ yield a morphism $Y \rightarrow \widetilde{Y}$ which is an eventual weak equivalence (starting from $k$ ). This yields a commutative square

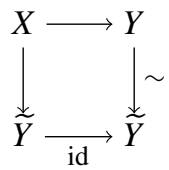

which proves (App2).

We define a functor $|-|: D K \rightarrow \mathbb{N}$ by sending $x \in D K$ to the smallest $k \in \mathbb{N}$ such that $x \in D^{(k)} K$. We call $|x|$ the filtration degree of $x$. Here, we do not consider any particular homotopical structure on $\mathbb{N}$ so $|-|$ is not a homotopical functor. We will be interested in the left Kan extension of a homotopical Reedy cofibrant diagram $X: D K \rightarrow \mathcal{C}$ along $|-|$. It can be computed as

$$
\left(\operatorname{Lan}_{|-|} X\right)_{k}=\operatorname{colim}_{D^{(k)} K} X
$$

We will denote $\left(\operatorname{Lan}_{|-|} X\right)_{k}$ by $\Phi^{(k)} X$ and when $k$ varies $\Phi^{(-)} X$ will stand for the resulting sequence $\mathbb{N} \rightarrow \mathcal{C}$. 
Just as colimits can be defined in terms of cones, left Kan extensions can be defined in terms of certain generalized cones. We describe such cones for Kan extensions along $|-|$. Let $D K \star_{|-|} \mathbb{N}$ denote the cograph (or collage) of $|-|$ defined as the category whose set of objects is the disjoint union of the sets of objects of $D K$ and $\mathbb{N}$ and

$$
\left(D K \star_{|-|} \mathbb{N}\right)(x, y)= \begin{cases}D K(x, y) & \text { when } x, y \in D K, \\ \mathbb{N}(x, y) & \text { when } x, y \in \mathbb{N}, \\ \mathbb{N}(|x|, y) & \text { when } x \in D K \text { and } y \in \mathbb{N}, \\ \varnothing & \text { otherwise. }\end{cases}
$$

Alternatively, $D K \star_{|-|} \mathbb{N}$ can be constructed as the pushout square

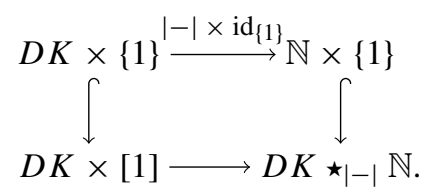

The left Kan extension of $X: D K \rightarrow \mathcal{C}$ along $|-|$ is nothing but an initial extension of $X$ to $D K \star_{|-|} \mathbb{N}$ so that morphisms $\Phi^{(-)} X \rightarrow Y$ in $\mathcal{C}^{\mathbb{N}}$ correspond to diagrams on $D K \star_{|-|} \mathbb{N}$ restricting to $X$ and $Y$ on $D K$ and $\mathbb{N}$ respectively. Such an extension of $X$ is a family of cones under the restrictions of $X$ to all $D^{(k)} K \mathrm{~s}$. We will compare them to extensions to $D\left(K^{\triangleright}\right)$ using a functor $p_{K}: D\left(K^{\triangleright}\right) \rightarrow D K \star_{|-|} \mathbb{N}$ defined as follows. Write an object of $D\left(K^{\triangleright}\right)$ as $x \star \varphi$ with $x \in D^{a} K$ and $\varphi \in D^{a}[0]$ and set

$$
p_{K}(x \star \varphi)= \begin{cases}|x \star \varphi| & \text { when } \varphi \in D[0], \\ x & \text { otherwise. }\end{cases}
$$

This allows us to state and prove a version of Lemma 4.2 for finitely cocomplete cofibration categories.

Lemma 5.10 Let $\mathcal{C}$ be a cofibration category, $K$ a finite simplicial set and $X: D K \rightarrow$ $\mathcal{C}$ a homotopical Reedy cofibrant diagram. Consider a morphism $f: \Phi^{(-)} X \rightarrow Y$ and the corresponding cone $\widetilde{T}: D K \star_{|-|} \mathbb{N} \rightarrow \mathcal{C}$. If $T$ is any Reedy cofibrant replacement of $p_{K}^{*} \widetilde{T}$ relative to $D K$ (i.e. $T$ is Reedy cofibrant and comes with a weak equivalence $T \stackrel{\sim}{\rightarrow} p_{K}^{*} \widetilde{T}$ that is an identity over $D K$, such a replacement exists by Lemma 1.9), then $f$ factors as

$$
\Phi^{(-)} X \rightarrow \Phi^{(-)} T \stackrel{\sim}{\rightarrow} Y
$$

where the latter morphism is an eventual weak equivalence (starting at $\operatorname{dim} K+1$ ).

Proof To verify that the above composite agrees with $f$ it suffices to check that at each level $k$ it agrees upon precomposition with $X_{x} \rightarrow \Phi^{(k)} X$ for all $x \in D^{(k)} K$. That's indeed the case since $T \mid D K=X$.

It remains to check that the latter morphism is an eventual weak equivalence. For $i \geq \operatorname{dim} K+1$ in the diagram 


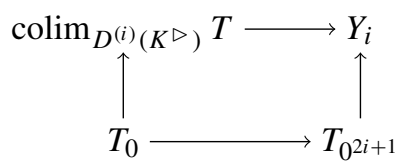

the left morphism is a weak equivalence by Lemma 5.8 and so is the right one since $T$ is a cofibrant replacement of $p_{K}^{*} \widetilde{T}$. The bottom morphism is a weak equivalence by the homotopical structure of $D\left(K^{\triangleright}\right)$ and therefore so is the the top one.

For every $m \geq 0$ each object of $D(K \star \Delta[m])$ can be uniquely written as $x \star \varphi$ with $x \in D^{a} K$ and $\varphi \in D^{a}[m]$. This yields a functor $r_{K}: D(K \star \Delta[m]) \rightarrow D^{a}[m]$ sending $x \star \varphi$ to $\varphi$ and to which we can associate the "filtered" left Kan extension functor

$$
\operatorname{Lan}_{r_{K}}^{\text {filt }}: \mathcal{C}_{\mathrm{R}}^{D(K \star \Delta[m])} \rightarrow\left(\mathcal{C}_{\mathrm{R}}^{\widetilde{\mathbb{N}}}\right)_{\mathrm{R}}^{D^{a}[m]}
$$

defined as $\left(\operatorname{Lan}_{r_{K}}^{\text {filt } X)_{\varphi}}=\Phi^{(-)} \varphi^{*} X\right.$ for $\varphi \in D^{a}[m]$. The functor $\operatorname{Lan}_{r_{K}}^{\text {filt }}$ is exact by [14, Theorem 9.4.3(1)]. Similarly we have

$$
\operatorname{Lan}_{s_{K}}^{\text {filt }}:\left(\mathcal{C}_{\mathrm{R}}^{\widetilde{\mathbb{N}}}\right)_{\mathrm{R}}^{D(K \star \partial \Delta[m])} \rightarrow\left(\mathcal{C}_{\mathrm{R}}^{\widetilde{\mathbb{N}}}\right)_{\mathrm{R}}^{D^{a} \partial \Delta[m]}
$$

where $s_{K}: D(K \star \partial \Delta[m]) \rightarrow D^{a} \partial \Delta[m]$ is a functor defined in the same way as $r_{K}$. We form pullbacks (the front and back squares of the cube)

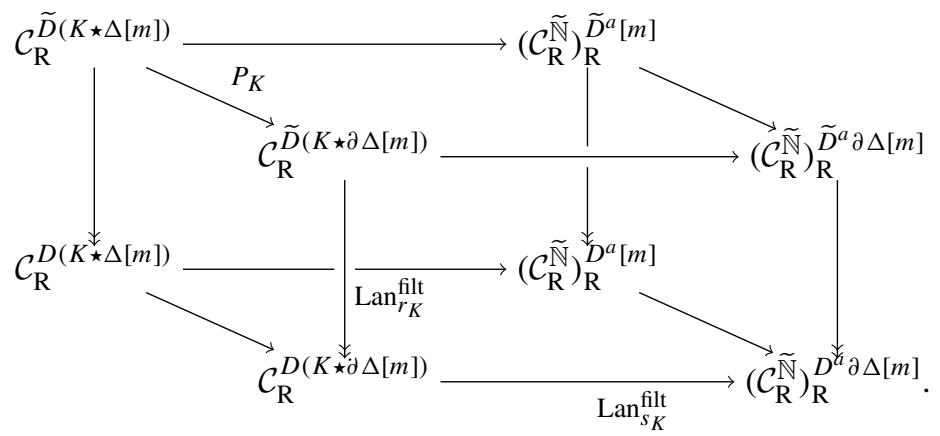

Observe that $\mathcal{C}^{\widetilde{D}(K \star \Delta[m])}$ and $\mathcal{C}^{\widetilde{D}(K \star \partial \Delta[m])}$ are just atomic notations for the pullbacks above, i.e. $\widetilde{D}(K \star \Delta[m])$ and $\widetilde{D}(K \star \partial \Delta[m])$ are $n o t$ homotopical categories for general $K$, although they will be interpreted as such when $K$ is a simplex.

The following is a finite variant of Lemma 4.5.

Lemma 5.11 The functor $P_{K}: \mathcal{C}_{\mathrm{R}}^{\widetilde{D}(K \star \Delta[m])} \rightarrow \mathcal{C}_{\mathrm{R}}^{\widetilde{D}(K \star \partial \Delta[m])}$ is an acyclic fibration for every finite simplicial set $K$.

Proof The proof is virtually identical to the proof of Lemma 4.5 except that now we do not consider the cases of coproducts and colimits of sequences of monomorphisms and we use Lemma 5.8 in the place of Lemma 4.1.

Finally, we can characterize colimits in $\mathrm{N}_{\mathrm{f}} \mathcal{C}$ in terms of homotopy colimits in $\mathcal{C}$ in a manner similar to Theorem 4.6. 
Theorem 5.12 Let $\mathcal{C}$ be cofibration category, $K$ a finite simplicial set. A cone $S: K^{\triangleright} \rightarrow \mathrm{N}_{\mathrm{f}} \mathcal{C}$ is universal if and only if the induced morphism

$$
\Phi^{(-)}(S \mid K) \rightarrow \Phi^{(-)} S
$$

is an eventual weak equivalence (where $S$ is seen as a homotopical Reedy cofibrant diagram $D\left(K^{\triangleright}\right) \rightarrow \mathcal{C}$ by Proposition 2.6). Such a cone exists under every diagram $K \rightarrow \mathrm{N}_{\mathrm{f}} \mathcal{C}$.

Proof The proof is almost identical to the proof of Theorem 4.6 except that we use Lemmas 5.8, 5.10 and 5.11 in the place of Lemmas 4.1, 4.2 and 4.5 respectively.

Acknowledgements This paper is based on a part of my thesis [19] which was written while I was a doctoral student in Bonn International Graduate School in Mathematics and, more specifically, Graduiertenkolleg 1150 "Homotopy and Cohomology" and International Max Planck Research School for Moduli Spaces. I want to thank everyone involved for creating an excellent working environment. I want to thank Clark Barwick, Bill Dwyer, André Joyal, Chris Kapulkin, Lennart Meier, Thomas Nikolaus, Chris SchommerPries, Peter Teichner and Marek Zawadowski for conversations on various topics which were very beneficial to my research. I am especially grateful to Viktoriya Ozornova and Irakli Patchkoria for reading an early draft of my thesis. Their feedback helped me make many improvements and avoid numerous errors. Above all, I want to express my gratitude to my supervisor Stefan Schwede whose expertise was always invaluable and without whose support this thesis could not have been written.

\section{References}

1. Avigad, J., Kapulkin, K., Lumsdaine, P.L.: Homotopy limits in type theory. Math. Struct. Comput. Sci. 25(5), 1040-1070 (2015)

2. Borceux, F.: Handbook of categorical algebra. 1. Encyclopedia of Mathematics and its Applications, vol. 50, Cambridge University Press, Cambridge. Basic category theory (1994)

3. Brown, K.S.: Abstract homotopy theory and generalized sheaf cohomology. Trans. Am. Math. Soc. 186, 419-458 (1973)

4. Cordier, J.M.: Sur la notion de diagramme homotopiquement cohérent. Cahiers Topologie Géom. Différentielle 23(1), 93-112 (1982) (French). Third Colloquium on Categories, Part VI (Amiens, 1980)

5. Dwyer, W.G., Kan, D.M.: Simplicial localizations of categories. J. Pure Appl. Algebra 17(3), 267-284 (1980)

6. Dwyer, W.G., Kan, D.M.: Calculating simplicial localizations. J. Pure Appl. Algebra 18(1), 17-35 (1980)

7. Gabriel, P., Zisman, M.: Calculus of Fractions and Homotopy Theory, Ergebnisse der Mathematik und ihrer Grenzgebiete, vol. 35. Springer-Verlag New York Inc, New York (1967)

8. Hovey, M.: Model categories, Mathematical Surveys and Monographs, vol. 63. American Mathematical Society, Providence, RI (1999)

9. Joyal, A.: Quasi-categories and Kan complexes. J. Pure Appl. Algebra 175(1-3), 207-222 (2002). doi:10.1016/S0022-4049(02)00135-4. Special volume celebrating the 70th birthday of Professor Max Kelly. MR1935979

10. Joyal, A.: The Theory of Quasi-Categories and its Applications. Quadern 45, Vol. II, Centre de Recerca Matemàtica Barcelona (2008)

11. Joyal, A., Tierney, M.: Quasi-categories vs Segal spaces. Categories in algebra, geometry and mathematical physics, Contemp. Math., vol. 431, pp. 277-326. Amer. Math. Soc., Providence, RI (2007)

12. Kapulkin, K.: Locally Cartesian Closed Quasicategories From Type Theory. arXiv:1507.02648

13. Kapulkin, K., Szumiło, K.: Quasicategories of frames of cofibration categories. Appl. Categ. Struct., 1-25 (2016)

14. Răadulescu-Banu, A.: Cofibrations in Homotopy Theory (2006). http://arxiv.org/abs/math/0610009v4 
15. Rezk, C.: A model for the homotopy theory of homotopy theory. Trans. Amer. Math. Soc. 353(3), 973-1007 (2001) (electronic)

16. Rourke, C.P., Sanderson, B.J.: $\triangle$-sets. I. Homotopy theory. Quart. J. Math. Oxford Ser. 22(2), 321-338 (1971)

17. Riehl, E., Verity, D.: The theory and practice of Reedy categories. Theory Appl. Categ. 29, 256-301 (2014)

18. Schwede, S.: The p-order of topological triangulated categories. J. Topol. 6(4), 868-914 (2013)

19. Szumiło, K.: Two Models for the Homotopy Theory of Cocomplete Homotopy Theories. Ph.D. Thesis, Rheinische Friedrich-Wilhelms-Universität Bonn (2014). http://hss.ulb.uni-bonn.de/2014/3692/3692. htm

20. Szumiło, K.: Two Models for the Homotopy Theory of Cocomplete Homotopy Theories (2014). arXiv: 1411.0303

21. Szumiło, K.: Homotopy theory of cofibration categories. Homol. Homotop. Appl (to appear)

22. Szumiło, K.: Homotopy theory of cocomplete quasicategories. Algebraic Geom Topol (to appear) 